

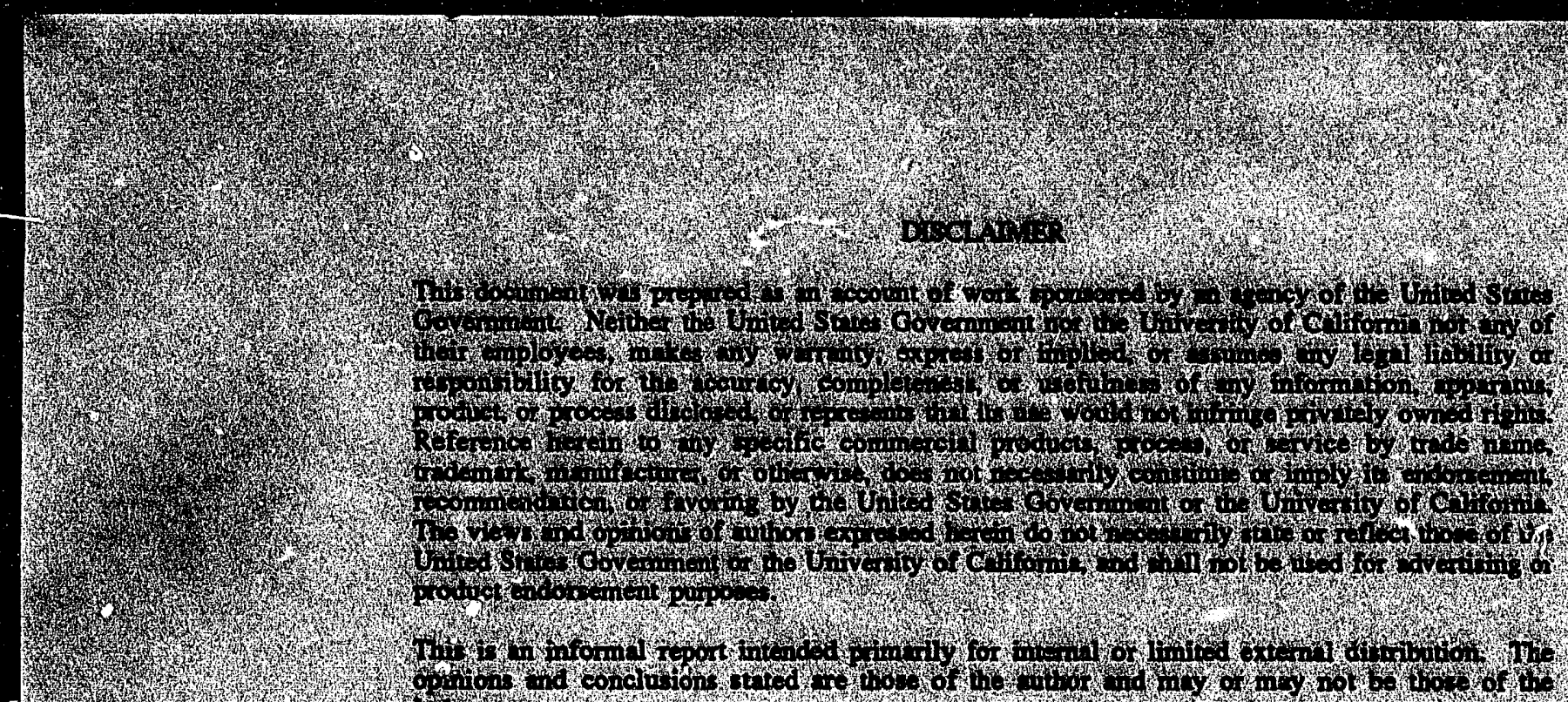

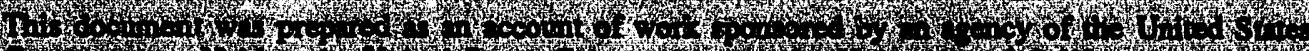

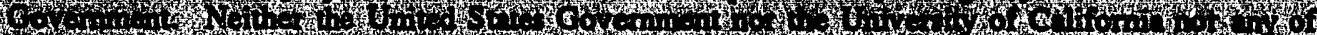

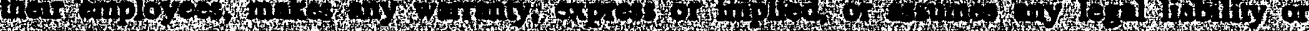

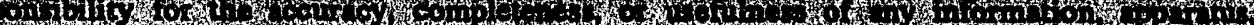
4.

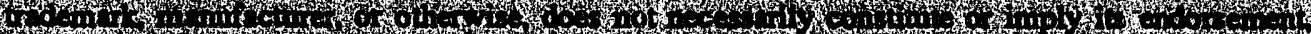
烈

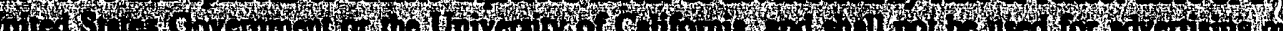

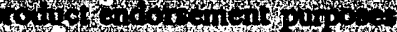

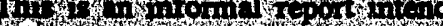
15.

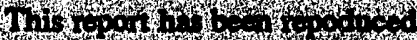
at

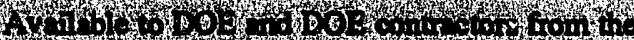

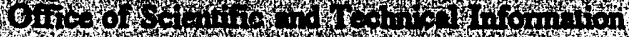

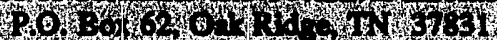

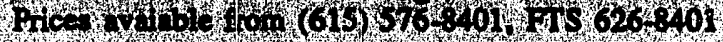

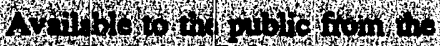

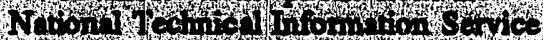

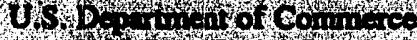

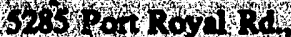

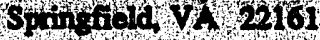




\section{ABSTRACT \\ A PROGRAM FOR PASSIVELY TRACKING A TARGET USING AN ARRAY OF SENSORS}

The objective of this document is to present: a program which passively tracks a target using an array of sensors. This program is available in MATLAB, version 3.5. The algorithm which is implemented consists of three main parts: time delay estimation, passive localization, and data post processing. Each of these parts are discussed, and the mathematical foundation for their solution given. Following this, the organization of the program is presented, and an example of its usage is given. 


\section{ACKNCWLEDGEMENTS}

I would like to thank James V. Candy, James A. Markevitch, and Larry C. $\mathrm{Ng}$ for the knowledge and guidance provided. This work was funded by $O$ Program. 


\section{TABLE OF CONTENTS}

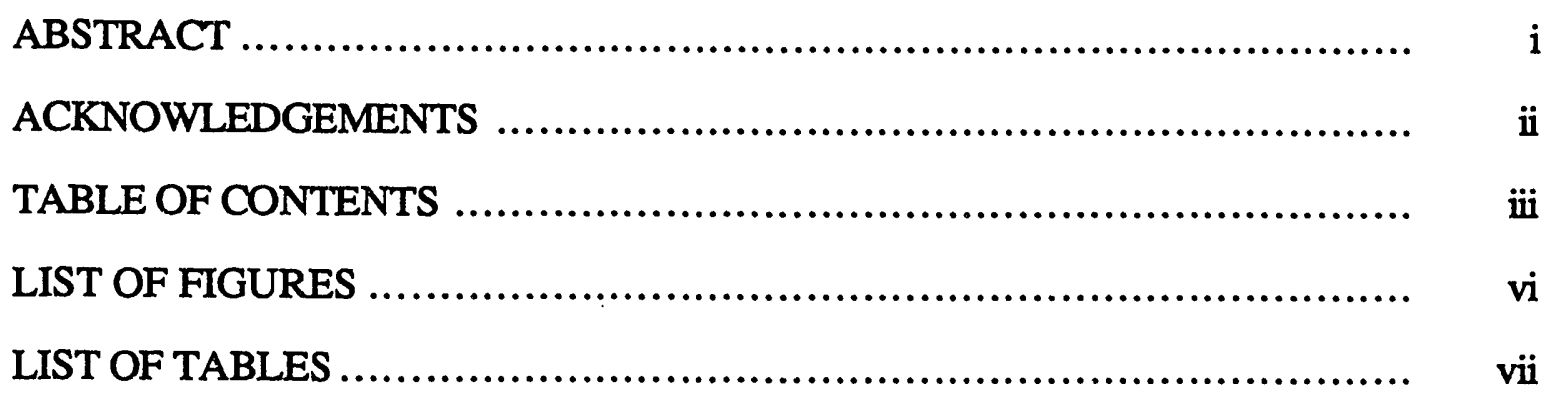

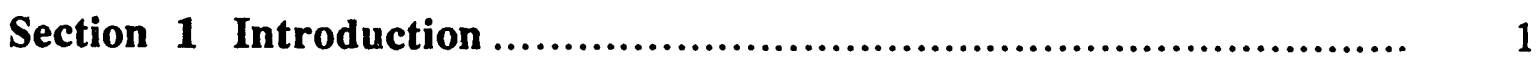

Section 2 Time Delay Estimation........................................ 3

2.1. Introduction ................................................ 3

2.2. Mathematical Theory ........................................ 3

2.2.1. Unity Gain Processor .............................. 5

2.2.2. Roth Processor......................................... 5

2.2.3. Smoothed Coherence Transform (SCOT) Processor...... 6

2.2.4. Phase Transform (PHAT) Processor................... 7

2.2.5. Maximum Likelihood (ML) Processor ................. 7

2.3. Summary ................................................... 9

Section 3 Passive Localization ........................................... 10

3.1. Introduction ............................................... 10

3.2. Mathematical Theory ......................................... 10

Section 4 Post Processing................................................... 14

4.1. Introduction ................................................. 14

4.2. Mathernatical Theory ............................................ 14 
Section 5 Program Description .......................................... 17

5.1. Introduction ................................................... 17

5.2. Program Organization .......................................... 17

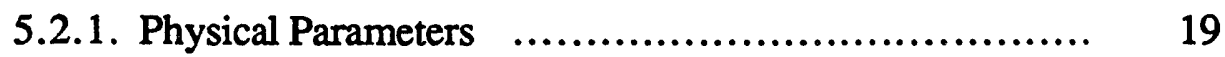

5.2.2. Sensor Signals ....................................... 20

5.2.2.1. Source Signal Characteristic Parameters ...... 21

5.2.2.2. Source Signal Trajectory Parameters ......... 22

5.2.3. Tracking Parameters .................................. 23

5.2.4. Tracking................................................. 24

5.2.5. Display .............................................. 24

5.3. Example Problem ........................................... 25

5.3.1. Problem Specifications ................................ 25

5.3.2. Programming Session ................................ 26

5.4. Additional Considerations ....................................... 34

5.5. Availability ................................................... 40

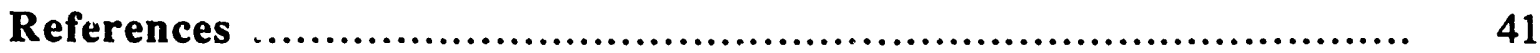




\section{LIST OF FIGURES}

\section{Section 1 Introduction}

1.1. Passive tracking block diagram ............................... 1

\section{Section 2 Time Delay Estimation}

2.1. Filtered cross-correlation

\section{Section 5 Program Description}

5.1. Flow diagram of program sequences

5.2. Sensor signals for the example problem

5.3. Time delay between sensor signals and the reference sensor for the example problem

5.4. Estimated and actual source trajectories for the example problem 


\section{LIST OF TABLES}

\section{Section 2 Time Delay Estimation}

2.1. Summary of time delay processors ..............................

\section{Section 5 Program Description}

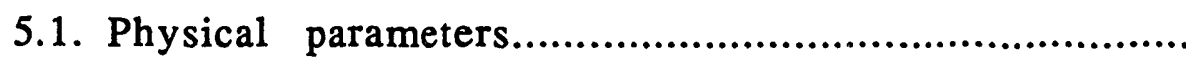

5.2. Source signal characteristic parameters for signals which are comprised of colored noise events

5.3. Source signal characteristic parameters for signals which are comprised of second order events

5.4. Source signal trajectory parameters

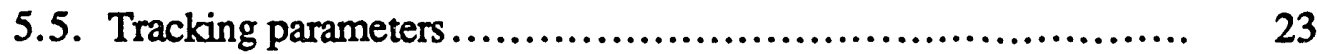

5.6. Variables which are saved into result file 


\section{Introduction}

\subsection{Introduction}

The problem of passively tracking a moving signal source has importance in a variety of applications such as radar, sonar, seismology, and radio astronomy. In many applications, only limited information is available about the signal source. For the algorithm discussed in this document, it will be assumed that only the signals which are detected by the sensors and the velocity of the source signal are known.

The problem of passively tracking a moving target using an array of sensors is shown below.

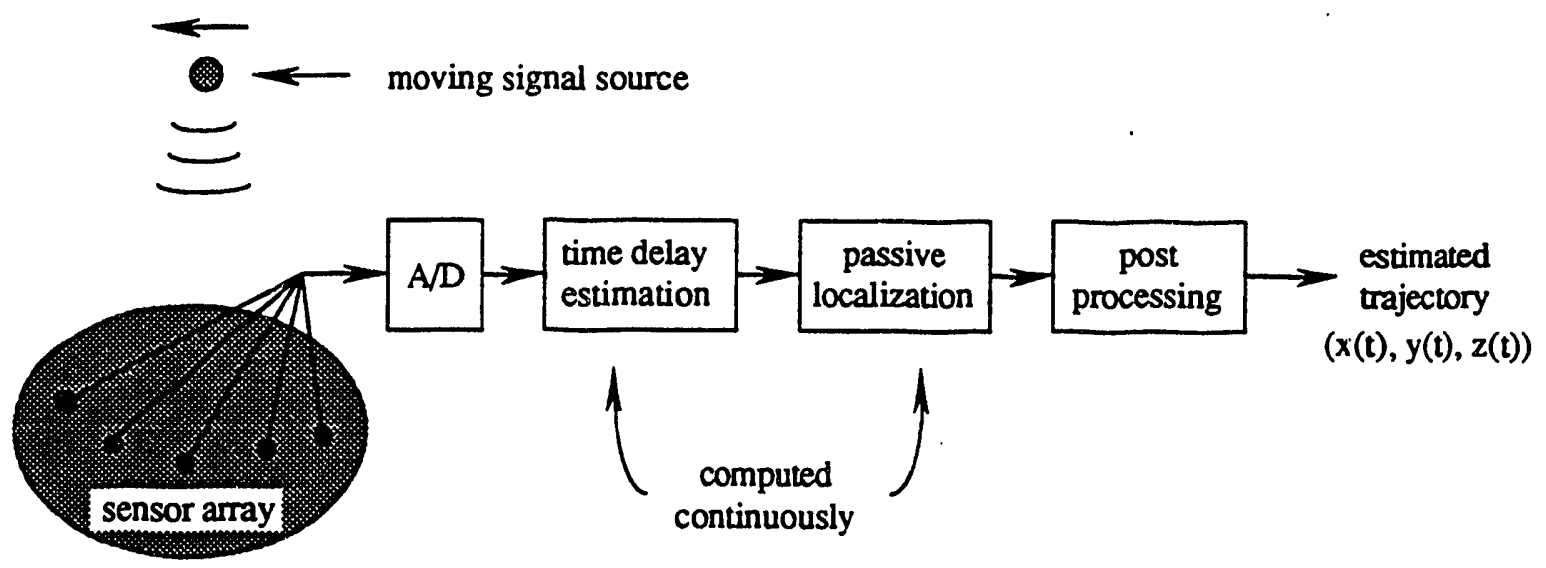

Fig. 1.1. Passive tracking block diagrami

In this figure, the signal source is moving in three dimensions. This motion can be specified by

$$
\begin{aligned}
& x(t)=f_{x}(t) \\
& y(t)=f_{y}(t) \\
& z(t)=f_{z}(t) .
\end{aligned}
$$

Each sensor is fixed in position and represented by a coordinate location, also in three dimensions. Since the sensor signals are continuous, they must be sampled before they are 
stored in monory. These signals can then be digitally processed to compute the source trajectory. This computation is accomplished in three steps. First, the time delays are computed relative to a reference sensor. Second, a passive localization algorithm uses the time delays, the sensor array geometry, and the signal velocity to compute the source location. These two steps are repeated over and over to generate a source trajectory. Lastly, the source locations from the previous two steps are processed to smooth the trajectories and interpolate intermediate points.

Sections 2 through 4 of this document discuss this algorithm in more detail. The second section describes the time delay estimation. In this section, the computation of the time delays utilizing the cross-correlation is discussed. In an effort to improve upon the accuracy of these estimates, a method of filiering the sensor signals prior to computing the cross-correlation is presented. The third section describes the passive localization algorithm which estimates the source location. This algorithm computes the least squares solution to a set of linear equations which are derived from the estimated time delays between the sensor signals, the signal velocity, and the sensor geometry. The fourth section describes post processing of the data. The purpose of this step is to decrease the variance of source locations; thereby improving the trajectories. This step also interpolates source locations between those computed by the passive localization algorithm. Lastly, the fifth section describes a computer program which can be used to study the effectiveness of this approach to particular problems. This program can also be used to study the effects various parameters have on the tracking ability of this algorithm. An example problem is included and results are given. 


\section{Time Delay Estimation}

\subsection{Introduction}

In this section, the computation of the time delays is discussed. Five techniques are presented which are based on finding the time at which the cross-correlations between two sensor signals peak. The mathematical theory for these techniques is given in section 2.2 , and a summary of them is given in section 2.3 .

\subsection{Mathematical Theory}

Consider the problem of estimating the delay between two sensor signals. These sampled signals can be modeled as

$$
\begin{aligned}
& x_{1}(n)=s_{1}(n)+v_{1}(n) \\
& x_{2}(n)=s_{2}(n)+v_{2}(n)=\alpha \cdot s_{1}(n-D)+v_{2}(n)
\end{aligned}
$$

where $s_{i}(n)(i=1$ and 2$)$ are the sampled and attenuated signals arriving at sensor $i$ due solely to the source, and $v_{1}(n)$ and $v_{2}(n)$ are noise. Furthermore, it is assumed that $s_{1}(n)$, $v_{1}(n)$, and $v_{2}(n)$ are uncorrelated, stationary, and have zero mean. Given these assumptions, the cross-correlation of $x_{1}(n)$ and $x_{2}(n)$ is given by

$$
R_{x_{1} x_{2}}(k)=R_{s_{1} s_{2}}(k)=\alpha \cdot E\left\{s_{1}(n) s_{1}(n-D+k)\right\}=\alpha \cdot R_{s_{1} s_{1}}(k-D)
$$

From the properties of auto-correlation,

$$
R_{s_{1} s_{1}}(l)=E\left\{s_{1}(n) s_{1}(n+1)\right\} \leq R_{s_{1} s_{1}}(0)
$$

Indicating that $R_{\mathbf{x}_{1} \mathbf{x}_{2}}(\mathrm{k})$ must peak at $\mathrm{k}$ equal to the time delay, $\mathrm{D}$. This is the simplest technique used to estimate the time delay. 
An alternative representation of Eqn. (2.3) is given by

$$
R_{\mathbf{x}_{1} \mathbf{X}_{2}}(k)=\alpha \cdot R_{S_{1} S_{1}}(k-D)=\alpha \cdot\left\{R_{S_{1} S_{1}}(k) * \delta(k-D)\right\}
$$

where * is the convolution operator, and $\delta$ is the delta function. From Eqn. (2.5), it is evident that $R_{\mathbf{x}_{1} \mathbf{x}_{2}}(\mathrm{k})$ can be interpreted as a "smeared" delta function. From the property of autocorrelations given by Eqn. (2.4), it appears from Eqns. (2.3) and (2.5) that fairly accurate estimates of the time delays are easily achieved. However, since only finite record lengths are available, the correlations given above can only be estimated, which in turn may lead to errors in computing the time delay. Additionally, $s_{1}(n), v_{1}(n)$, and $v_{2}(n)$ may not be uncorrelated.

One way to alleviate these effects, thereby increasing time delay estimate accuracy, is to filter $x_{1}(n)$ and $x_{2}(n)$ prior to computing the cross-correlations. In doing so, sharper peaks may result at the time delay, which in turn may lead to more accurate time delay estimates. This process is shown below.

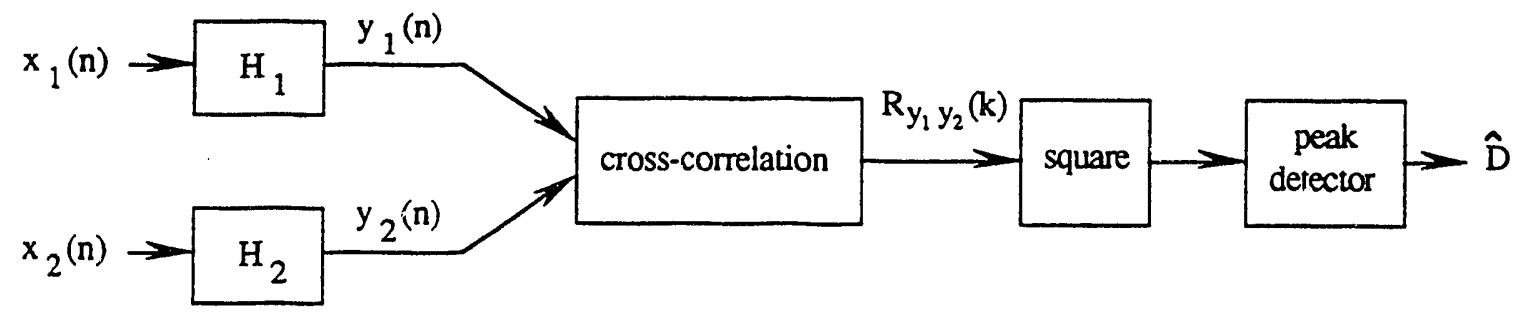

Figure 2.1. Filtered cross-correlation

The filtered cross-correlation from Fig. 2.1 is given by

$$
R_{y_{1} y_{2}}(k)=E\left\{y_{1}(n) y_{2}(n+k)\right\}=\operatorname{iDFT}\left\{S_{y_{1} y_{2}}(\omega)\right\}
$$

where $S_{y_{1} y_{2}}(\omega)$ is the cross-power spectrum of $y_{1}(n)$ and $y_{2}(n)$, and iDFT is the inverse discrete Fourier transform [1]. In order to determine the time delays between $x_{1}(n)$ and 
$x_{2}(n)$, the relationship between these signals and $R_{y_{1} y_{2}}(k)$ is necessary. From the properties of random linear systems [1], the cross-power spectral relationship is given by

$$
S_{y_{1} y_{2}}(\omega)=H_{1}(\omega) H_{2}^{*}(\omega) S_{x_{1} x_{2}}(\omega)
$$

Substituting Eqn. (2.7) into Eqn. (2.6) yields

$$
R_{y_{1} y_{2}}(k)=\operatorname{iDFT}\left\{\Psi(\omega) S_{x_{1} x_{2}}(\omega)\right\}
$$

where

$$
\Psi(\omega)=\mathrm{H}_{1}\left(\mathrm{H}_{2}^{*}(\omega)\right.
$$

Therefore, $\Psi(\omega)$ should be chosen in such a way so as to "sharpen" the peaks of the crosscorielations at the time delay, leading to more accurate time delay estimates. Much effort has been spent to study the problem of selecting processors, $\Psi(\omega)$, which will optimize performance criteria. A brief discussion of five processors follows in sections 2.2.1 to 2.2.5. For more detailed discussions refer to [1-9].

\subsubsection{Unity Gain Processor}

The unity gain processor [1-4] is given by

$$
\Psi(\omega)=1
$$

This processor does not provide filtering of $x_{1}(n)$ and $x_{2}(n)$ prior to the cross-correlation computation. It is therefore the simplest time delay technique.

\subsubsection{Roth Processor}

The Roth processor $[4,6]$ is given by

$$
\Psi(\omega)=\frac{1}{S_{x_{1} x_{1}}(\omega)}
$$


In order to better interpret the effect of this processor, the power spectrum of $x_{1}(n)$ can be specified in terms of the power spectrum of $s_{1}(n)$ and $v_{1}(n)$ with the aid of Eqn. (2.1). Holding to the earlier assumption that $s_{1}(n)$ and $v_{1}(n)$ are uncorrelated, this yields

$$
\Psi(\omega)=\frac{1}{S_{S_{1} S_{1}}(\omega)+S_{v_{1} v_{1}}(\omega)}
$$

Substituting Eqn. (2.12) irto Eqn. (2.8) results in

$$
R_{y_{1} y_{2}}(k)=\operatorname{iDFT}\left\{\frac{S_{x_{1} x_{2}}(\omega)}{S_{s_{2} s_{1}}(\omega)+S_{v_{1} v_{1}}(\omega)}\right\}
$$

From this equation, the effect of the Roth processor becomes apparent. At frequencies where the power of $v_{1}(n)$ is large relative to the power of $s_{1}(n)$ (low $S N R$ ), $S_{y_{1} y_{2}}(\omega)$ is suppressed. Therefore at these frequencies, $R_{y_{1} y_{2}}(k)$ contains smaller frequency components than $R_{x_{1} x_{2}}(k)$.

\subsubsection{Smoothed Coherence Transform (SCOT) Processor}

The smoothed coherence transform processor (SCOT) $[4,5,7,8]$ is given by

$$
\Psi(\omega)=\frac{1}{\sqrt{S_{x_{1} x_{1}}(\omega) S_{x_{2} x_{2}}(\omega)}} .
$$

The Roth processor suppresses frequencies where $S_{v_{1} v_{1}}(\omega)$ is large, but it doesn't account for frequencies where $S_{v_{2} v_{2}}(\omega)$ is large. To remedy this problem, the SCOT processor takes the power spectral density of both $v_{1}(n)$ and $v_{2}(n)$ into account. With the aid of Eqn. (2.9) and Fig. 2.1, it is evident that the time delay computation can be viewed as two filters followed by a cross correlator, where the filters are given by

$$
H_{i}(\omega)=\frac{1}{\sqrt{S_{x_{i} x_{i}}(\omega)}} \quad(i=1,2) \text {. }
$$


The effect of these filters is to whiten the data prior to computing the cross-correlation, thereby sharpening the function peak.

\subsubsection{Phase Transform (PHAT) Processor}

The PHAT processor $[4,8]$ is given by

$$
\Psi(\omega)=\frac{1}{\left|S_{x_{1} x_{2}}(\omega)\right|} .
$$

Substituting Eqn. (2.16) into Eqn. (2.8) results in

$$
R_{y_{1} y_{2}}(k)=\operatorname{iDFT}\left\{\frac{S_{x_{1} x_{2}}(\omega)}{\left|S_{x_{1} x_{2}}(\omega)\right|}\right\} \text {. }
$$

With the aid of Eqn. (2.5), $\mathrm{S}_{\mathrm{x}_{1} \mathrm{x}_{2}}(\omega)$ can be given as

$$
S_{x_{1} x_{2}}(\omega)=D F T\left\{R_{x_{1} x_{2}}(k)\right\}=\alpha \cdot S_{S_{1} S_{1}}(\omega) \cdot e^{-j \omega D}=\left|S_{x_{1} x_{2}}(\omega)\right| \cdot e^{-j \omega D} .
$$

$S_{S_{1} S_{1}}(\omega)$ is real-valued since $R_{S_{1} S_{1}}(k)$ is an even function [1]. Substituting Eqn. (2.18) into Eqn. (2.17) yields

$$
R_{y_{1} y_{2}}(k)=\operatorname{iDFT}\left\{e^{-j \omega D}\right\}=\delta(k-D) .
$$

From Eqn. (2.19) it appears as if the PHAT processor eliminates the "smearing" in the cross correlations. However, since only finite record lengths are available, the spectral densities can only be estimated. Additionally, the sensor signal noise may not be uncorrelated. For these reasons, the cross-correlation will not be a perfect delta function, but will exhibit "smearing".

\subsubsection{Maximum Likelihood (ML) Processor}

The maximum likelihood processor is given by 


$$
\Psi(\omega)=\frac{|\gamma|^{2}}{\left(1-|\gamma|^{2}\right)\left|S_{\mathrm{x}_{1} \mathrm{x}_{2}}(\omega)\right|},
$$

where $|\gamma|^{2}$ is the magnitude square coherence specified by

$$
|y|^{2}=\frac{\left|S_{x_{1} x_{2}}(\omega)\right|^{2}}{S_{x_{1} x_{1}}(\omega) S_{x_{2} x_{2}}(\omega)}
$$

This processor attempts to maximize the probability of obtaining discrete Fourier coefficients for $x_{1}(n)$ and $x_{2}(n)$ given the power and cross power spectral densities. This is summarized below. For more detailed discussions refer to $[4,5,9]$.

The discrete Fourier coefficients of $x_{1}(n)$ and $x_{2}(n)$ are given by

$$
\begin{aligned}
& a(m)=\frac{1}{N} \sum_{n} x_{1}(n) e^{-j \Omega_{m^{n}}} \\
& b(m)=\frac{1}{N} \sum_{n} x_{2}(n) e^{-j \Omega_{m^{n}}} .
\end{aligned}
$$

where there are $N$ points in the signals, $n$ is the summation over $N$, and $\Omega_{m}=\frac{2 \pi m}{N}$. If it is assumed that $x_{1}(n)$ and $x_{2}(n)$ are gaussian, then the discrete coefficients are also gaussian since they are linear transformations of $x_{1}(n)$ and $x_{2}(n)$. Given this assumption and the earlier assumption of $s_{1}(n), v_{1}(n)$, and $v_{2}(n)$ being uncorrelated, the probability of discrete Fourier coefficients can be determined as a function of spectral densities, time delay, and signal attenuation. This can be represented mathematically by

$$
p(a(m), b(m))=f\left(\alpha, S_{s_{1} S_{1}}(\omega), S_{v_{1} v_{1}}(\omega), S_{v_{2} v_{2}}(\omega), D\right) \text {. }
$$

The estimated time delay between $x_{1}(n)$ and $x_{2}(n)$ is the value of $D$ which maximizes Eqn. (2.24). This is equivalent to selecting the delay where $R_{y_{1} y_{2}}(k)$ peaks given the processor of Eqns. (2.20) and (2.21). Substituting these equations into Eqn. (2.8) yields

$$
\mathbf{R}_{\mathrm{y}_{1} \mathrm{y}_{2}}(\mathrm{k})=\operatorname{iDFT}\left\{\frac{|\gamma|^{2}}{\left(1-|\gamma|^{2}\right)} \frac{S_{\mathrm{x}_{1} \mathbf{x}_{2}}(\omega)}{\left|S_{\mathrm{x}_{1} \mathbf{x}_{2}}(\omega)\right|}\right\}=\operatorname{iDFT}\left\{\frac{|\gamma|^{2}}{\left(1-|\gamma|^{2}\right)} \mathrm{e}^{-\mathrm{j} \omega \mathrm{D}}\right\}
$$


This processor is similar to the PHAT processor. However, it weights the phase given in Eqn. (2.19) by a function of the squared coherence. At frequencies where $x_{1}(n)$ and $x_{2}(n)$ are highly correlated, the weighting is large, and at frequencies where these signals are less correlated, the weighting is smaller.

\subsection{Summary}

A summary of five different time delay processors is given in Table 2.1.

\begin{tabular}{|c|c|}
\hline $\begin{array}{l}\text { Processor } \\
\text { Name }\end{array}$ & $\Psi(\omega)$ \\
\hline Unity Gain & 1 \\
\hline \multirow{2}{*}{ Roth } & 1 \\
\hline & $S_{x_{1} x_{1}}(\omega)$ \\
\hline \multirow{2}{*}{ SCOT } & 1 \\
\hline & $\sqrt{S_{x_{1} x_{1}}(\omega) S_{x_{2} x_{2}}(\omega)}$ \\
\hline \multirow{2}{*}{ PHAT } & 1 \\
\hline & $\left|S_{x_{1} x_{2}}(\omega)\right|$ \\
\hline \multirow{2}{*}{ ML } & $|\gamma(\omega)|^{2}$ \\
\hline & $\left|S_{x_{1} x_{2}}(\omega)\right|\left[1-|\gamma(\omega)|^{2}\right]$ \\
\hline
\end{tabular}

Table. 2.1. Summary of time delay processors

This completes the discussion of time delay estimators. The next section will discuss the passive localization algorithm. 


\section{Passive Localization}

\subsection{Introduction}

In this section, the passive localization method is discussed. This algorithm computes the least squares solution to a set of linear equations. If there are " $N$ " sensor signals, there will be "N-1" linear equations. These equations are derived from the estimated time delays between a reference sensor signal and the remaining "N-1" sensor signals, the signal velocity, and the sensor geometry. For this method, there must be at least " $\mathrm{N}$ " sensors, where " $\mathrm{N}$ " is greater than the number of spatial dimensions plus one. The discussion below assumes there are three spatial dimensions. Therefore, there must be at least five sensors. The mathematical theory for this method is given in section 3.2. For a more detailed discussion refer to [10].

\subsection{Mathematical Theory}

Let the location of the sensors and the source be specified in three dimensions as $\mathrm{q}_{\mathrm{i}}=$ $\left[x_{i}, y_{i}, z_{i}\right]^{T}$ and $q_{s}=\left[x_{s}, y_{s}, z_{s}\right]^{T}$, respectively. Furthermore, assume tha: the reference for these coordinate locations is the origin of the three dimensional space $[0,0,0]$. With this notation the range difference between two sensors " $i$ " and " $j$ " is given by

$$
r_{i j}=\left\|q_{i}-q_{s}\right\|-\left\|q_{j}-q_{s}\right\|
$$

where $\mathrm{r}_{\mathrm{ij}}$ can be computed simply by multiplying the time delay between sensors " $\mathrm{i}$ " and " $\mathrm{j}$ " by the signal velocity. Using this definition of range difference, $\left\|q_{i}-q_{s}\right\|^{2}$ is given by

$$
\left\|q_{i}-q_{s}\right\|^{2}=\left(r_{i j}+\left\|q_{j}-q_{s}\right\|\right)^{2}=r_{i j}^{2}+2\left\|q_{j}-q_{s}\right\| r_{i j}+\left\|q_{j}-q_{s}\right\|^{2}
$$

Additionally, $\left\|\mathrm{q}_{\mathrm{i}}-\mathrm{q}_{\mathrm{s}}\right\|^{2}$ can also be given by

$$
\left\|q_{i}-q_{s}\right\|^{2}=\left\|q_{i}\right\|^{2}+\left\|q_{s}\right\|^{2}-2 q_{i}^{T} q_{s}
$$


Equating the right hand side of Eqns. (3.2) and (3.3) yields

$$
2 q_{i}{ }^{T} q_{s}=\left\|q_{i j}\right\|^{2}+\left\|q_{s}\right\|^{2}-r_{i j}{ }^{2}-2\left\|q_{j}-q_{s}\right\| r_{i j}-\left\|q_{j}-q_{s}\right\|^{2} .
$$

Setting $i=j$, and noting that $r_{i j}=0$ for $i=j$, results in

$$
2 q_{j}{ }^{T} q_{s}=\left\|q_{j}\right\|^{2}+\left\|q_{s}\right\|^{2}-\left\|q_{j}-q_{s}\right\|^{2} .
$$

Subtracting Eqn. (3.5) from Eqn. (3.4) yields

$$
2\left(q_{i}-q_{j}\right)^{T} q_{s}=\left(\left\|q_{i}\right\|^{2}-\left\|q_{j}\right\|^{2}\right)-r_{i j}{ }^{2}-2\left\|q_{j}-q_{s}\right\| r_{i j}
$$

This equation can be written in matrix form as given by

$$
S_{j} x_{s}=\mu_{j}-\left\|q_{j}-q_{s}\right\| p_{j},
$$

where

$$
\begin{aligned}
& s_{j}=\left[\begin{array}{ccc}
\left(x_{1}-x_{j}\right) & \left(y_{1}-y_{j}\right) & \left(z_{1}-z_{j}\right) \\
\vdots & \vdots & \vdots \\
\left(x_{j-1}-x_{j}\right) & \left(y_{j-1}-y_{j}\right) & \left(z_{j-1}-z_{j}\right) \\
\left(x_{j+1}-x_{j}\right) & \left(y_{j+1}-y_{j}\right) & \left(z_{j+1}-z_{j}\right) \\
\vdots & \vdots & \vdots \\
\left(x_{N}-x_{j}\right) & \left(y_{N}-y_{j}\right) & \left(z_{N}-z_{j}\right)
\end{array}\right] \\
& \mu_{j}=\frac{1}{2}\left[\begin{array}{ccc}
\left\|q_{1}\right\|^{2} & -\left\|q_{j}\right\|^{2} & -r_{i j}^{2} \\
\vdots & \vdots & \vdots \\
\left\|q_{j-1}\right\|^{2} & -\left\|q_{j}\right\|^{2} & -r_{j-1 j}^{2} \\
\left\|q_{j+1}\right\|^{2} & -\left\|q_{j}\right\|^{2} & -r_{j+1 j}^{2} \\
\vdots & \vdots & \vdots \\
\left\|q_{N}\right\|^{2} & -\left\|q_{j}\right\|^{2} & -\dot{r}_{N j}^{2}
\end{array}\right] \text {, and }
\end{aligned}
$$




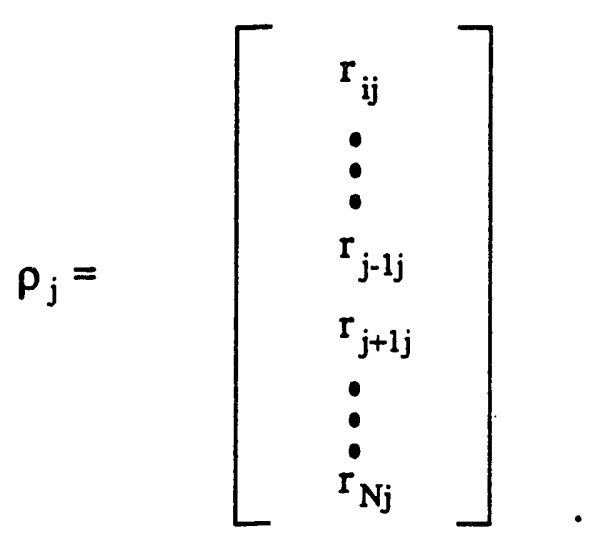

Sensor signal $\mathrm{jl}$ is the reference in these equations . All quantities in Eqn. (3.7) are known except for $x_{s}$ and $\left\|q_{j}-q_{s}\right\|$. To simplify the solution of this equation, it is multiplied by a matrix $M_{j}$, where $M_{j}$ is in the null-space of $\rho_{j}$. One such matrix is given by

$$
M_{j}=V_{k}{ }^{T} D_{j} \text {, }
$$

where $D_{j}$ is a diagonal matrix given by

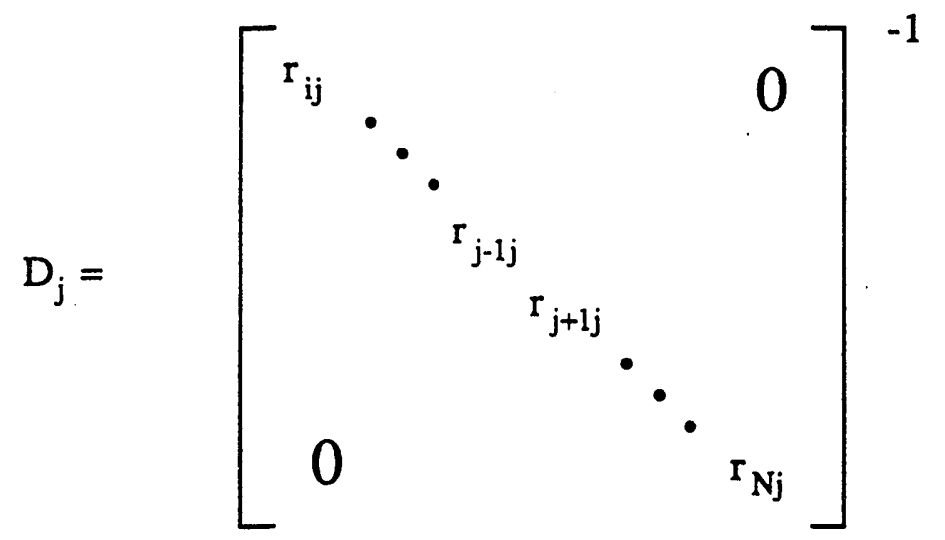

$\mathrm{V}_{\mathrm{k}} \mathrm{T}^{\mathrm{T}}$ is computed using the singular value decomposition as

$$
\left(I-Z^{k}\right)=\left[U_{k}, u_{k}\right]\left[\begin{array}{cccc}
n_{1}^{k} & & & 0 \\
& \ddots & & \\
& & n_{N-2}^{k} & \\
0 & & & 0
\end{array}\right]\left[V_{k}, v_{k}\right]^{T}
$$


where I is the "N-1" $x$ "N-1" identity matrix and $Z^{k}$ is the "N-1" $x$ "N-1" circular shift matrix given by

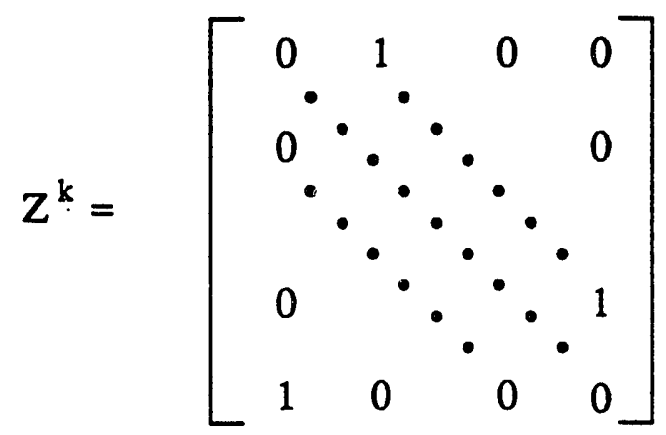

Multiplying Eqn. (3.7) by $M_{j}$ yields

$$
M_{j} S_{j} x_{s}=M_{j} \mu_{j} .
$$

A least squares technique can be used to solve for $\mathbf{x}_{\mathbf{s}}$. A closed-form solution is given by

$$
x_{s}=\left(S_{j}{ }^{T} M_{j}{ }^{T} M_{j} S_{j}\right)^{-1} S_{j}{ }^{T} M_{j}{ }^{T} M_{j} \mu_{j}
$$

In order for $\mathrm{M}_{\mathrm{j}} \mathrm{S}_{\mathrm{j}}$ to be nonsingular, it is necessary (but not sufficient) that the number of rows of $S_{j}$ be greater than the number of columns. Therefore to obtain a unique solution, the number of sensors, "N", must be greater than the number of dimensions plus one.

This completes the discussion of the passive localization. The next section will discuss the post processing of the estimated source locations. 


\section{Post Processing}

\subsection{Introduction}

In this section, the post processing of the computed trajectory points is discussed. The post processing can be broken into two steps. First, the source locations computed by the passive localization algorithm are smoothed. This is accomplished by fitting a second order polynomial to the data. Second, the source locations are interpolated at times intermediate to those computed by the passive localization. The mathematical theory is given in section 4.2 .

\subsection{Mathematical Theory}

This algorithm is given the computed source location and the corresponding time from the passive localization algorithm. These points will be represented by $x_{i}\left(t_{i}\right), y_{i}\left(t_{i}\right)$, and $z_{i}\left(t_{i}\right)$. The polynomial curve fitting algorithm fits second degree polynomials through these sets of data points. These polynomials are given by

$$
\begin{aligned}
& x(t)=c_{x 2} t^{2}+c_{x 1} t+c_{x 0}, \\
& y(t)=c_{y 2} t^{2}+c_{y 1} t+c_{y 0}, \text { and } \\
& z(t)=c_{z 2} t^{2}+c_{z 1} t+c_{z 0} .
\end{aligned}
$$

Substituting $\mathrm{x}_{\mathrm{i}}\left(\mathrm{t}_{\mathrm{i}}\right), \mathrm{y}_{\mathrm{i}}\left(\mathrm{t}_{\mathrm{i}}\right)$, and $\mathrm{z}_{\mathrm{i}}\left(\mathrm{t}_{\mathrm{i}}\right)$ into Eqns. (4.1) to (4.3) yields the linear equations

$$
\begin{aligned}
& x_{i}\left(t_{i}\right)=c_{x 2} t_{i}^{2}+c_{x 1} t_{i}+c_{x 0}, \\
& y_{i}\left(t_{i}\right)=c_{y 2} t_{i}^{2}+c_{y 1} t_{i}+c_{y 0}, \text { and } \\
& z_{i}\left(t_{i}\right)=c_{z 2} t_{i}^{2}+c_{z 1} t_{i}+c_{z 0} .
\end{aligned}
$$

These can be represented in matrix form as 


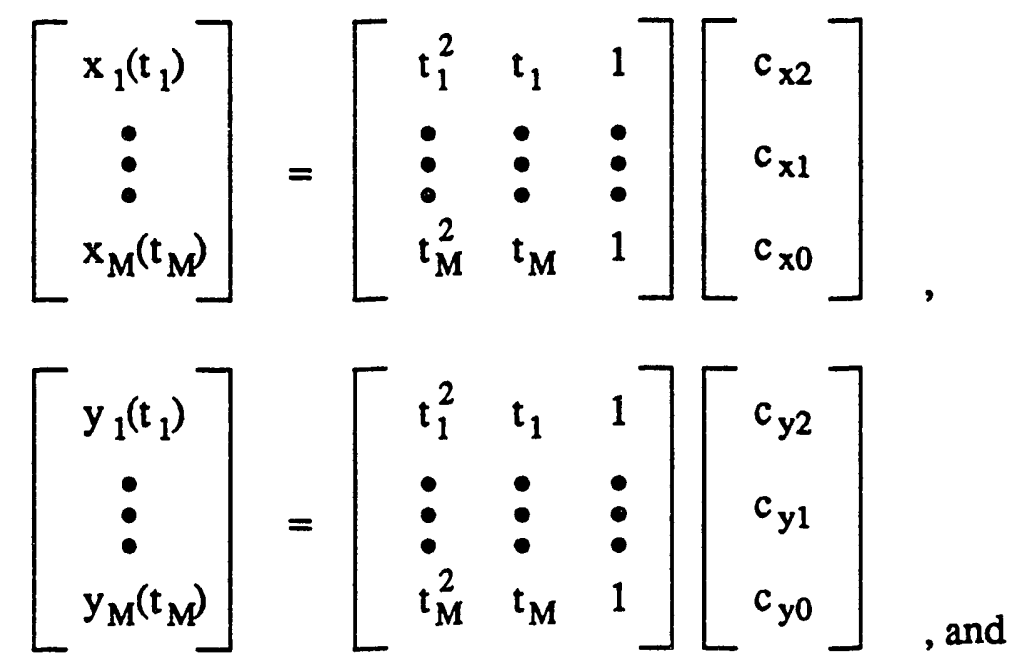

$\left[\begin{array}{c}\mathrm{z}_{1}\left(\mathrm{t}_{1}\right) \\ \vdots \\ \mathrm{z}_{M}\left(\mathrm{t}_{M}\right)\end{array}\right]=\left[\begin{array}{ccc}\mathrm{t}_{1}^{2} & \mathrm{t}_{1} & 1 \\ \vdots & \vdots & \vdots \\ \mathrm{t}_{M}^{2} & \mathrm{t}_{M} & 1\end{array}\right]\left[\begin{array}{c}\mathrm{c}_{\mathrm{z} 2} \\ \mathrm{c}_{\mathrm{z} 1} \\ \mathrm{c}_{\mathrm{z} 0}\end{array}\right]$

A least squares technique can be used to solve for the coefficients in Eqns. (4.7) to (4.9). By defining Eqns. (4.7) to (4.9) as

$$
\begin{aligned}
& \bar{x}=T_{x} \overline{c_{x}} \\
& \bar{y}=T_{y} \bar{c}_{y}, \text { and } \\
& \bar{z}=T_{z} \bar{c}_{z},
\end{aligned}
$$

the least squares solution is given by

$$
\begin{aligned}
& \overline{c_{x}}=\left(T_{x}{ }^{\prime} T_{x}\right)^{-1} T_{x}{ }^{\prime} \bar{x} \\
& \overline{c_{y}}=\left(T_{y}{ }^{\prime} T_{y}\right)^{-1} T_{y}{ }^{\prime} \bar{y} \\
& \overline{c_{z}}=\left(T_{z}{ }^{\prime} T_{z}\right)^{-1} T_{z}{ }^{\prime} \bar{z}
\end{aligned}
$$

Once the coefficients are computed, the points intermediate to $t_{i}$ are interpolated directly from the polynomials, as given by Eqns. (4.1) to (4.3). 
This completes the discussion of the post-processing. The next section will present a computer program which can be used to study the performance of the passive tracking algorithm. 


\section{Program Description}

\subsection{Introduction}

This section presents a computer program which can be used to study the performance of the passive tracking algorithm to particular problems. It also may be used to study the effects various parameters have on the tracking ability of this algorithm. This menu-driven program is available in MATLAB, version 3.5. Section 5.2 presents the program organization. Following this section, an example is given in section 5.3. Section 5.4 presents additional considerations relating to data files. Finally, section 5.5 provides information regarding the availablity of this program.

\subsection{Program Organization}

The following steps must be taken prior to applying the tracking algorithm.

1. Physical parameters must be initialized.

2. Sensor signals must be initialized.

3. Tracking parameters must be initialized.

The physical parameters consist of the sensor array geometry, the signal velocity, the signal attenuation constant, the sampling period, and the variance of the sensor signal noise. These parameters may be specified by the user or loaded in from a file. After the physical parameters are initialized, the user must initialize the sensor signal. These signals can also be specified by the user or loaded in from a file. If the user wishes to specify these signals, additional parameters must be initialized. These include the source signal characteristic parameters and the source trajectory parameters. Once these are specified, the sensor signals can be generated. Signals which are generated in this manner will be referred to as simulated sensor signals. In contrast, sensor signals which are obtained in a real environment will be referred to as real sensor signals. Both types of sensor signals may be 
loaded from a file. After the sensor signals are initialized, the user can specify the tracking algorithm parameters. These parameters consist of the reference sensor, the period of trajectory point computations, the window length of the signal used to estimate the time delays, and the time delay processor. Following this, all necessary variable have been initialized, and the tracking can be performed. After the tracking has been performed, the user may go back and change any parameters before performing the tracking again. However, because the steps listed above are not decoupled, there are restrictions as to the sequence of steps. In addition to the steps given above, the user may display the sensor signals, the relative time delays, or the estimated and actual source trajectories. All possible sequences of steps are shown in Fig. 5.1. If the user should violate this sequence, an error message will be displayed. The blocks shown in Fig. 5.1 are presented in more detail in sections 5.2.1 to 5.2.5.

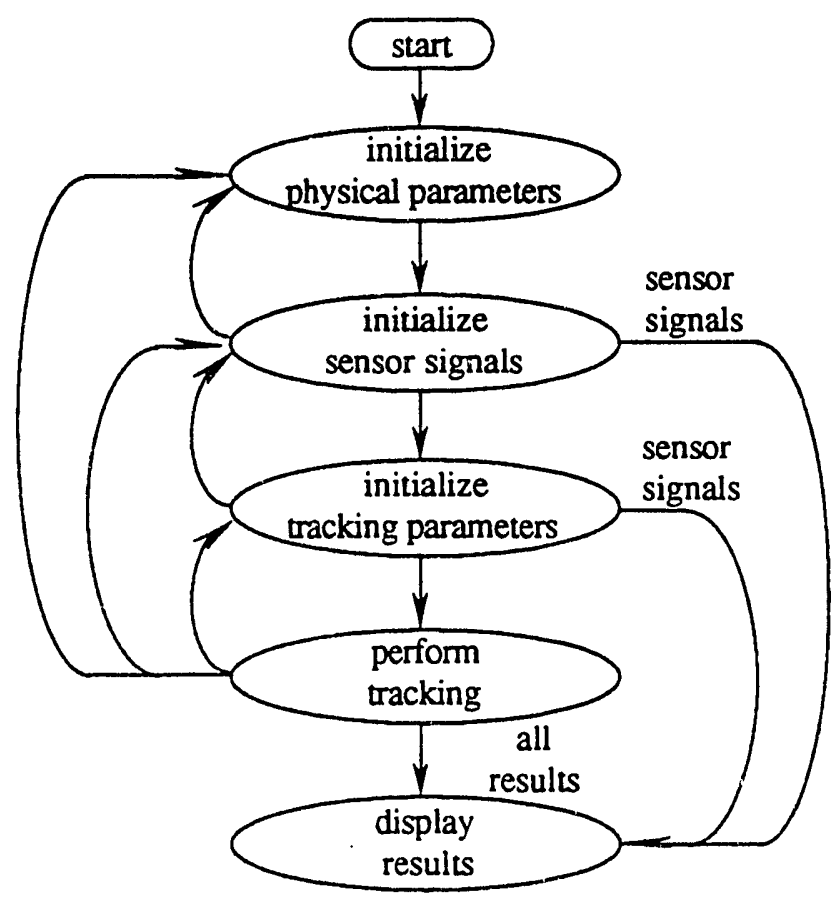

Fig. 5.1. Flow diagram of program sequences 


\subsubsection{Physical Parameters}

The physical parameters are specifications which are independent of the sensor signal characteristics. As stated above, these parameters may be specified by the user or loaded from a file. These parameters are defined below, in Table 5.1.

\begin{tabular}{|c|c|}
\hline $\begin{array}{l}\text { Physical Parameter } \\
\text { Name }\end{array}$ & Description \\
\hline Number of sensors & The number of sensors in the array (at least five). \\
\hline Sensor coordinates & $\begin{array}{l}\text { The three dimensional }(x, y, z) \text { sensor coordinates } \\
\text { (meters). }\end{array}$ \\
\hline Signal velocity & The velocity of the source signal (meters/second). \\
\hline $\begin{array}{l}\text { Source signal } \\
\text { attenuation constant }\end{array}$ & $\begin{array}{l}\text { The constant which determines the signal mag- } \\
\text { nitude at the sensors (meters). }\end{array}$ \\
\hline Sampling period & $\begin{array}{l}\text { The sampling period of the sensor signals } \\
\text { (seconds). }\end{array}$ \\
\hline $\begin{array}{l}\text { Variance of sensor } \\
\text { signal noise }\end{array}$ & $\begin{array}{l}\text { The variance of normally distributed, zero mean } \\
\text { noise which is added to the sensor signals. }\end{array}$ \\
\hline
\end{tabular}

Table 5.1. Physical parameters

All of these parameters self explanatory with the exception of the source signal attenuation constant. This constant determines the attenuation of the source signal as it arrives at each sensor. The attenuation is given by

$$
\text { Signal attenuation }=\frac{\text { Source signal attenuation constant }}{\text { distance }} \text { between source and sensor } .
$$

The way in which this quantity is used in the generation of the sensor signals is explained in section 5.2.2. 
It should also be noted that the variance of the sensor signal noise is only utilized it the sensor signals are to be specified (generated). If the sensor signals are loaded, this parameter is not used.

\subsubsection{Sensor Signals}

Similar to the physical parameters, the sensor signals may be specified by the user or loaded from a file. If the user chooses to specify these signals, the source signal characteristic parameters and the source signal trajectory parameters must be initialized. These are discussed in section 5.2.2.1 and 5.2.2.2, respectively. Once these parameters are initialized, the sensor signals can be generated. The generation of these signals is a two step process. First, the source signal is generated from the source signal characteristic parameters. Next, the sensor signals can be generated from the source signal, the physical parameters, and the source trajectory. These signals are generated by summing up the delayed and attenuated impulses which make up the source signal. The delay is determined by the distance between the source and the sensor at the time of the impulse and the signal velocity, and the attenuation is determined by Eqn. 5.1. Due to movement of the source, the sensor signals will be distorted with respect to one another. If the user wishes to load simulated sensor signals from a file, care must be taken to ensure that the sensor signals were generated using the physical parameters as specified for the current problem. This is due to the fact that the generation of the sensor signals is dependent upon the physical parameters. Likewise, if the user wishes to load real sensor signals from a file, care must be taken to ensure that the sensor signals were gathered using the physical parameters as specified for the current problem. The signal processing is identical for simulated and real data. Their only difference is in the display of the trajectories. If simulated data is processed, the actual trajectories are displayed along with the estimated trajectories. If real data is processed, the actual trajectories are unknown, and therefore only estimated trajectories are displayed. 


\subsubsection{Source Signal Characteristic Parameters}

The source signal consists of events which are attenuated and delayed version of a characteristic signal. All the events in a signal have identical characteristics, with the exception of their power. They represent echoes in the signal. Additionally, these events may be either colored noise or second order in nature.

The colored noise events are normally distributed, zero mean noise which is filtered. The characteristics which describe such a source signal are given in Table 5.2.

\begin{tabular}{|c|l|}
\hline $\begin{array}{c}\text { "Colored Noise" Source Signal } \\
\text { Characteristic Parameters }\end{array}$ & \multicolumn{1}{|c|}{ Description } \\
\hline $\begin{array}{c}\text { Low cutoff } \\
\text { frequency }\end{array}$ & $\begin{array}{l}\text { The low cutoff frequency of the bandpass filter } \\
\text { which filters the events (Hertz). }\end{array}$ \\
\hline $\begin{array}{c}\text { High cutoff } \\
\text { frequency }\end{array}$ & $\begin{array}{l}\text { The high cutoff frequency of the bandpass filter } \\
\text { which filters the events (Hertz). }\end{array}$ \\
\hline $\begin{array}{c}\text { Bandpass } \\
\text { filter order }\end{array}$ & The order of the bandpass filter. \\
\hline $\begin{array}{c}\text { Length } \\
\text { of signal }\end{array}$ & The length of the source signal (seconds). \\
\hline $\begin{array}{c}\text { Number } \\
\text { of events }\end{array}$ & $\begin{array}{l}\text { The number of colored noise events in the source } \\
\text { signal. }\end{array}$ \\
\hline $\begin{array}{c}\text { Length } \\
\text { of events }\end{array}$ & The length of the events (seconds). \\
\hline $\begin{array}{c}\text { Time } \\
\text { of events }\end{array}$ & The time at which each event occurs (seconds). \\
\hline $\begin{array}{c}\text { Power } \\
\text { of events }\end{array}$ & The average power of each events (dBs). \\
\hline $\begin{array}{c}\text { Variance of source } \\
\text { signal noise }\end{array}$ & $\begin{array}{l}\text { The variance of normally distributed, zero mean } \\
\text { noise which is added to the source signal. }\end{array}$ \\
\hline
\end{tabular}

Table. 5.2. Source signal characteristic parameters for signals which are comprised of colored noise events 
A signal which consists of second order events is generated by passing delayed and attenuated impulses through a second order system. The characteristics which describe this type of source signal are given in Table 5.3.

\begin{tabular}{|c|c|}
\hline $\begin{array}{l}\text { "Second Order" Source Signal } \\
\text { Characteristic Parameters }\end{array}$ & Description \\
\hline $\begin{array}{l}\text { Damping } \\
\text { ratio }\end{array}$ & The damping ratio of the second order system. \\
\hline $\begin{array}{l}\text { Natural } \\
\text { frequency }\end{array}$ & $\begin{array}{l}\text { The natural frequency of the second order system } \\
\text { (Hertz). }\end{array}$ \\
\hline $\begin{array}{l}\text { Length } \\
\text { of signal }\end{array}$ & The length of the source signal (seconds). \\
\hline $\begin{array}{l}\text { Number } \\
\text { of events }\end{array}$ & $\begin{array}{l}\text { The number of second order events in the source } \\
\text { signal. }\end{array}$ \\
\hline $\begin{array}{l}\text { Time } \\
\text { of events }\end{array}$ & The time at which each event occurs (seconds). \\
\hline $\begin{array}{l}\text { Power } \\
\text { of events }\end{array}$ & $\begin{array}{l}\text { The total power of each events which is computed } \\
\text { over the length of the signal }(\mathrm{dBs}) \text {. }\end{array}$ \\
\hline $\begin{array}{l}\text { Variance of source } \\
\text { signal noise }\end{array}$ & $\begin{array}{l}\text { The variance of normally distributed, zero mean } \\
\text { noise which is added to the source signal. }\end{array}$ \\
\hline
\end{tabular}

Table 5.3. Source signal characteristic parameters for signals which are comprised of second order events

It should be noted that the power of events for the two types of signals is computed differently.

\subsubsection{Source Signal Trajectory Parameters}

Before the sensor signal can be generated, the source trajectory must be initialized. This trajectory is specified by the parameters given in Table 5.4. 


\begin{tabular}{|c|c|}
\hline $\begin{array}{c}\text { Source Signal } \\
\text { Trajectory Parameters }\end{array}$ & \multicolumn{1}{|c|}{ Description } \\
\hline \hline $\begin{array}{c}\text { X-plane trajectory } \\
\text { coefficients }\end{array}$ & $\begin{array}{l}\text { Coefficients } a_{2}, a_{1}, \text { and } a_{0}, \text { where } \\
x(t)=a_{2} t^{2}+a_{1} t+a_{0}\end{array}$ \\
\hline $\begin{array}{c}\text { Y-plane trajectory } \\
\text { coefficients }\end{array}$ & $\begin{array}{l}\text { Coefficients } b_{2}, b_{1}, \text { and } b_{0}, \text { where } \\
y(t)=b_{2} t^{2}+b_{1} t+b_{0}\end{array}$ \\
\hline $\begin{array}{c}\text { Z-plane trajectory } \\
\text { coefficients }\end{array}$ & $\begin{array}{l}\text { Coefficients } c_{2}, c_{1}, \text { and } c_{0}, \text { where } \\
z(t)=c_{2} t^{2}+c_{1} t+c_{0}\end{array}$ \\
\hline
\end{tabular}

Table. 5.4. Source signal trajectory parameters

\subsubsection{Tracking Parameters}

After the sensor signals have been initialized, the user may initialize the tracking parameters. These variables, which effect the passive tracking algorithm, are given in Table 5.5.

\begin{tabular}{|c|l|}
\hline Tracking Parameters & \multicolumn{1}{|c|}{ Description } \\
\hline $\begin{array}{c}\text { Reference } \\
\text { sensor }\end{array}$ & $\begin{array}{l}\text { The sensor signal to which all other time delays are } \\
\text { computed relative to. }\end{array}$ \\
\hline $\begin{array}{c}\text { Period of trajectory } \\
\text { point computations }\end{array}$ & $\begin{array}{l}\text { The period between the estimated trajectory points } \\
\text { (seconds). }\end{array}$ \\
\hline $\begin{array}{c}\text { Window length } \\
\text { (fixed windows) }\end{array}$ & $\begin{array}{l}\text { The length of the sensor signal sections which are } \\
\text { used to estimate the time delays (seconds). }\end{array}$ \\
\hline $\begin{array}{c}\text { Initial window length } \\
\text { (adaptive windows) }\end{array}$ & $\begin{array}{l}\text { The length of the sensor signal sections which is } \\
\text { used to estimate the first set of time delays (seconds). }\end{array}$ \\
\hline $\begin{array}{c}\text { Minimum window length } \\
\text { (adaptive windows) }\end{array}$ & $\begin{array}{l}\text { The minimum length of the sensor signal sections } \\
\text { which are used to estimate the time delays (seconds). }\end{array}$ \\
\hline $\begin{array}{c}\text { Time delay } \\
\text { processor }\end{array}$ & $\begin{array}{l}\text { The processor used to compute the time delay (unity } \\
\text { gain, Roth, SCOT, PHAT, or ML). }\end{array}$ \\
\hline
\end{tabular}

Table. 5.5. Tracking parameters 
For the tracking parameters, two different window length methods can be chosen. The first uses a fixed window length. This method uses the same length of data to compute the time delays for every trajectory point computation. The second uses an adaptive window length. For this method a variable length of data is used to compute the time delays for every trajectory point computation. The length, in points, is determined as the smallest number which is a power of two and is larger than eight times the largest relative delay computed in the previous computation. To ensure that this number isn't too small, a minimum window length is specified. If the computed window length is smaller than this value, the minimum window length of data is used to compute the time delays. In order to increase the speed of the tracking algorithm, the user may wish to chose window lengths which result in a power of two (points) when divided by the sampling period.

\subsubsection{Tracking}

When all the variables described above are initialized, the passive tracking algorithm can be applied. This algorithm computes the estimated time delays and the estimated source trajectories. These results may be saved into a file which can be used as input into additional signal processing algorithms, such as Kalman Filters. The variable which are saved are described in section 5.4 .

\subsubsection{Display}

The user may display the sensor signals, the estimated time delays, or the estimated and actual source trajectory. The steps in which these results can be viewed are given in Fig. 5.1. 


\subsection{Example Problem}

In this section, an example will be presented. Section 5.3 .1 will specify the parameters for this particular problem. Following this, a session is given to demonstrate the use of this program in section 5.3.2.

\subsubsection{Problem Specifications}

The following example, which processes simulated sensor signals, is a hypothetical one, presented only to demonstrate the use of this program. The parameters for this example are given below.

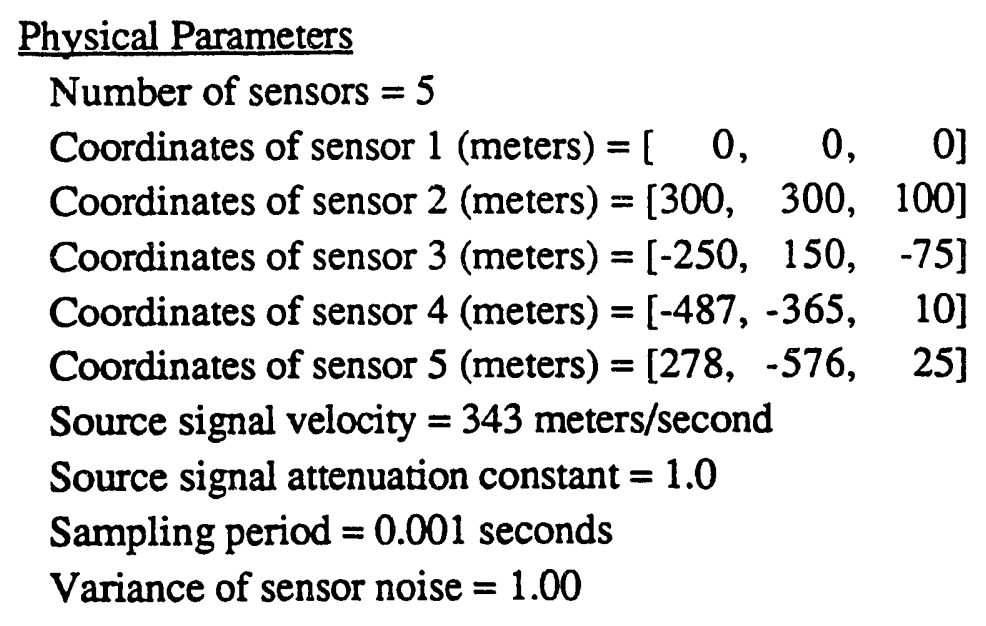

\section{Sensor Signal - Source Signal Characteristic Parameters}

Event type $=$ colored noise

Low cutoff frequency $=25 \mathrm{Hertz}$

High cutoff frequency $=250 \mathrm{Hertz}$

Bandpass filter order $=4$

Length of signal $=16.0$ seconds

Number of events $=1$

Length of events $=16.0$ seconds

Time of events $=0.0$ seconds

Power of events $=160 \mathrm{dBs}$

Variance of signal noise $=10.0$ 
Sensor Signal - Trajectory Parameters

$X$-plane trajectory coefficients $\left(\mathrm{m} / \mathrm{s}^{2}, \mathrm{~m} / \mathrm{s}, \mathrm{m}\right)=[0,40,0]$

Y-plane trajectory coefficients $\left(\mathrm{m} / \mathrm{s}^{2}, \mathrm{~m} / \mathrm{s}, \mathrm{m}\right)=[0,40,0]$

Z-plane trajectory coefficients $\left(\mathrm{m} / \mathrm{s}^{2}, \mathrm{~m} / \mathrm{s}, \mathrm{m}\right)=[5,0,15000]$

Tracking Parameters

Reference sensor $=1$

Period of trajectory point computations $=1.0$ seconds

Window length method $=$ adaptive

Initial window length $=1.024$ seconds

Minimum window length $=0.512$ seconds

Time delay processor $=$ unity gain

\subsubsection{Programming Session}

Following is the programming session. The program is started by typing "track". To aid in the use of this program, a flow diagram of the menu structure is given in Appendix A. The input which is typed by the user is italicized.

$»$ track

Main Menu

1) Initialize physical parameters

2) Initialize sensor signals

3) Initialize tracking parameters

4) Perform tracking

5) Display results

6) Exit

Select a menu number: 1

Physical Parameters Menu

1) Load physical parameters

2) Specify physical parameters

3) Goto main menu

Select a menu number: 2 
Enter the number of sensors (at least five): 5

Enter the coordinates of sensor 1 (meters) : $[0,0,0]$

Enter the coordinates of sensor 2 (meters) : [300,300,100]

Enter the coordinates of sensor 3 (meters) : $[-250,150,-75]$

Enter the coordinates of sensor 4 (meters) : $[-487,-365,10]$

Enter the coordinates of sensor 5 (meters) : [278,-576,25]

Enter the signal velocity (meters/second): 343

Enter the source signal attenuation constant (meters): 1.0

Enter the sampling period (seconds): 0.001

Enter the variance of the sensor signal noise: 1.0

Do you want to save these parameters to a file $[\mathrm{y} / \mathrm{n}]$ ? $y$

Enter the parameter file name: para_example

----- Physical Parameters Menu -----

1) Load physical parameters

2) Specify physical parameters

3) Goto main menu

Select a menu number: 3

----- Main Menu -----

1) Initialize physical parameters

2) Initialize sensor signals

3) Initialize tracking parameters

4) Perform tracking

5) Display results

6) Exit

Select a menu number: 2

Sensor Signal Menu --.--

1) Load sensor signals

2) Specify sensor signals

3) Goto main menu

Select a menu number: 2 
----- Simulate Sensor Signal Menu --.--

1) Specify source signal characteristics

2) Specify source trajectory

3) Generate sensor signals

4) Goto sensor signal menu

Select a menu number: 1

-.--- Event Type --.--

1) Colored noise events

2) Second order events

Select a menu number: 1

Enter the low cutoff frequency (Hertz): 25

Enter the high cutoff frequency (Hertz): 250

Enter the bandpass filter order: 4

Enter the length of the signal (seconds): 16.0

Enter the number of events: 1

Enter the length of the events (seconds): 16.0

Enter the time of events (seconds): 0.0

Enter the signal power of the events (dBs): 160

Enter the variance of the source signal noise: 10.0

----- Simulate Sensor Signal Menu ---.-

1) Specify source signal characteristics

2) Specify source trajectory

3) Generate sensor signals

4) Goto sensor signal menu

Select a menu number: 2

The trajectories are computed as follows, where distance is in meters and time in seconds:

$x(t)=a 2 * t^{*} t+a 1 * t+a 0$
$y(t)=b 2 * t * t+b 1 * t+b 0$
$z(t)=c 2 * t * t+c 1 * t+c 0$ 
Enter the X-plane trajectory coefficients [a2, a1, a0]: $[0,40,0]$

Enter the Y-plane trajectory coefficients [b2, b1, b0]: $[0,40,0]$

Enter the Z-plane trajectory coefficients [c2, c1, c0]: $[5,0,15000]$

----- Simulate Sensor Signal Menu -..--

1) Specify source signal characteristics

2) Specify source trajectory

3) Generate sensor signals

4) Goto sensor signal menu

Select a menu number: 3

The sensor signals are being generated ...

The sensor signals have been generated.

Do you want to save these sensor signals to a file $[\mathrm{y} / \mathrm{n}]$ ? $y$

Enter the sensor signal file name: sig_example

----- Simulate Sensor Signal Menu -----

1) Specify source signal characteristics

2) Specify source trajectory

3) Generate sensor signals

4) Goto sensor signal menu

Select a menu number: 4

Sensor Signal Menu

1) Load sensor signals

2) Specify sensor signals

3) Goto main menu

Select a menu number: 3

Main Menu

1) Initialize physical parameters

2) Initialize sensor signals

3) Initialize tracking parameters

4) Perform tracking

5) Display results

6) Exit

Select a menu number: 5 
----- Display Menu -.-.-

1) Sensor signals

2) Time delay estimates

3) Source trajectories

4) Goto main menu

Select a menu number: 1

Enter the sensor number: 1

(Sensor signal 1, shown in Fig. 5.2, appears in the graph window) Another plot $(\mathrm{y} / \mathrm{n}) ? \mathrm{y}$

Enter the sensor number: 2

(Sensor signal 2, shown in Fig. 5.2, appears in the graph window) Another plot $(y / n) ? y$

Enter the sensor number: 3

(Sensor signal 3, shown in Fig. 5.2, appears; in the graph window) Another plot $(\mathrm{y} / \mathrm{n}) ? \mathrm{y}$

Enter the sensor number: 4

(Sensor signal 4, shown in Fig. 5.2, appears in the graph window) Another plot $(y / n) ? y$

Enter the sensor number: 5

(Sensor signal 5, shown in Fig. 5.2, appears in the graph window) Another plot $(\mathrm{y} / \mathrm{n}) ? n$

----- Display Menu --.--

1) Sensor signals

2) Time delay estimation

3) Source trajectory

4) Goto main menu

Select a menu number: 4

----- Main Menu -.---

1) Initialize physical pararneters

2) Initialize sensor signals

3) Initialize tracking parameters

4) Perform tracking

5) Display results

6) Exit

Select a menu number: 3 
Enter the reference sensor: 1

Enter the period of trajectory point computations (seconds): 1.0

Select a window length method -----

1) Fixed window length

2) Adaptive window length

Select a menu number: 2

Enter the initial window length (seconds): 1.024

Enter the minimum window length (seconds): 0.512

----- Select a time delay processor --.--

1) Unity gain

2) Roth impulse response

3) Smoothed coherence transform (SCOT)

4) Phase transform (PHAT)

5) Maximum likelihood (ML)

Select a menu number: 1

\section{Main Menu}

1) Initialize physical parameters

2) Initialize sensor signals

3) Initialize tracking parameters

4) Perform tracking

5) Display results

6) Exit

Select a menu number: 4

The estimated relative delay vectors in seconds:

$\begin{array}{lllllll}\text { At time }= & 0.51 \text { seconds: } & 0.000 & -0.276 & 0.227 & 0.010 & -0.032 \\ \text { At time }= & 1.51 \text { seconds: } & 0.000 & -0.278 & 0.228 & 0.019 & -0.030 \\ \text { At time }= & 2.51 \text { seconds: } & 0.000 & -0.287 & 0.228 & 0.025 & -0.028 \\ \text { At time }= & 3.51 \text { seconds: } & 0.000 & -0.287 & 0.229 & 0.041 & -0.026 \\ \text { At time }= & 4.51 \text { seconds: } & 0.000 & -0.291 & 0.230 & 0.037 & -0.024 \\ \text { At time }= & 5.51 \text { seconds: } & 0.000 & -0.296 & 0.230 & 0.042 & -0.022 \\ \text { At time }= & 6.51 \text { seconds: } & 0.000 & -0.302 & 0.231 & 0.040 & -0.020 \\ \text { At time }= & 7.51 \text { seconds: } & 0.000 & -0.306 & 0.232 & 0.052 & -0.019 \\ \text { At time }= & 8.51 \text { seconds: } & 0.000 & -0.309 & 0.232 & 0.052 & -0.017 \\ \text { At time }= & 9.51 \text { seconds: } & 0.000 & -0.313 & 0.233 & 0.062 & -0.015 \\ \text { At time }= & 10.51 \text { seconds: } & 0.000 & -0.316 & 0.233 & 0.066 & -0.014 \\ \text { At time }= & 11.51 \text { seconds: } & 0.000 & -0.319 & 0.234 & 0.066 & -0.012\end{array}$




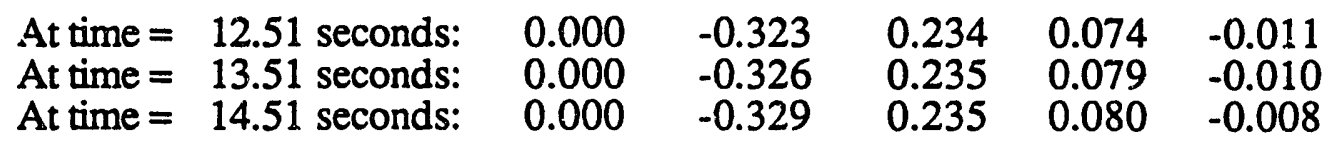

The estimated source locations in meters:

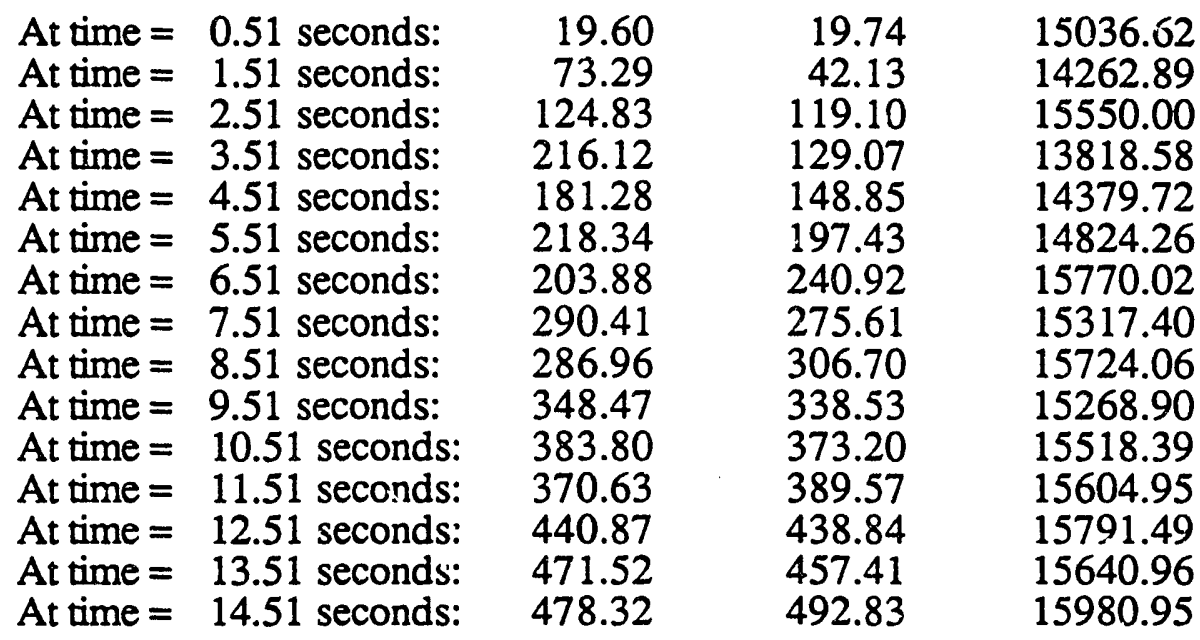

Do you want to save these results to a file $[\mathrm{y} / \mathrm{n}]$ ? $y$

Enter the result file name: res_example

Main Menu

1) Initialize physical parameters

2) Initialize sensor signals

3) Initialize tracking parameters

4) Perform tracking

5) Display results

6) Exit

Select a menu number: 5

----- Display Menu -----

1) Sensor signals

2) Time delay estimates

3) Source trajectories

4) Goto main menu

Select a menu number: 2 
Enter the sensor number (excluding reference) : 2

(The relative delay for sensor 2, shown in Fig. 5.3, appears in the graph window) Another plot $(\mathrm{y} / \mathrm{n}) ? \mathrm{y}$

Enter the sensor number (excluding reference) : 3

(The relative delay for sensor 3, shown in Fig. 5.3, appears in the graph window) Another plot $(\mathrm{y} / \mathrm{n}) ? y$

Enter the sensor number (excluding reference) : 4

(The relative delay for sensor 4, shown in Fig. 5.3, appears in the graph window) Another plot $(\mathrm{y} / \mathrm{n})$ ? $y$

Enter the sensor number (excluding reference) : 5

(The relative delay for sensor 5, shown in Fig. 5.3, appears in the graph window) Another plot $(\mathrm{y} / \mathrm{n}) ? n$

-..-- Display Menu --.--

1) Sensor signals

2) Time delay estimates

3) Source trajectories

4) Goto main menu

Select a nenu number. 3

(The X-plane trajectories, shown in Fig. 5.4, appear in the graph window) Hit any key to continue ...

(The Y-plane trajectories, shown in Fig. 5.4, appear in the graph window) Hit any key to continue ...

(The Z-plane trajectories, shown in Fig. 5.4, appear in the graph window)

...-. Display Menu -...-

1) Sensor signals

2) Time delay estimates

3) Source trajectories

4) Goto main menu

Select a menu number: 4 
----- Main Menu -.--.

1) Initialize physical parameters

2) Initialize sensor signals

3) Initialize tracking parameters

4) Perform tracking

5) Display results

6) Exit

Select a menu number: 6

This concludes the example.

For this particular problem, the passive tracking algorithm performed fairly well. However, this may not always be the case. As previously stated, the sensor signals are distorted relative to one another due to Doppler effects. This signal distortion causes time delay estimate errors which in turn cause source localization errors. Therefore, this algorithm may $\mathbf{p}^{\prime}$ :form well only for cases wh sre this distortion is small.

\subsection{Additional Considerations}

A couple points relating to the internal workings of this program are worth noting.

First, the sensor signal files may contain simulated or real data. The signal processing is identical for both data types. Their only difference is in the display of the trajectories. If simulated data is processed, the actual trajectories are displayed along with the estimated trajectories. If real data is processed, the actual trajectories are unknown, and therefore only estimated trajectories are displayed. Due to this difference, the sensor signal files must contain a variable, referred to as "Data_Type", which indicates the type of data. If this data represents real sensor signals, this variable should be 1 , and if this data represents simulated sensor signals, this variable should be 2 . Additionally, if this data represents simulated sensor signals, the actual trajectory coefficients, which were used to generate these signals, are stored in variables "X_Coef", "Y_Coef , and "Z_Coef". 

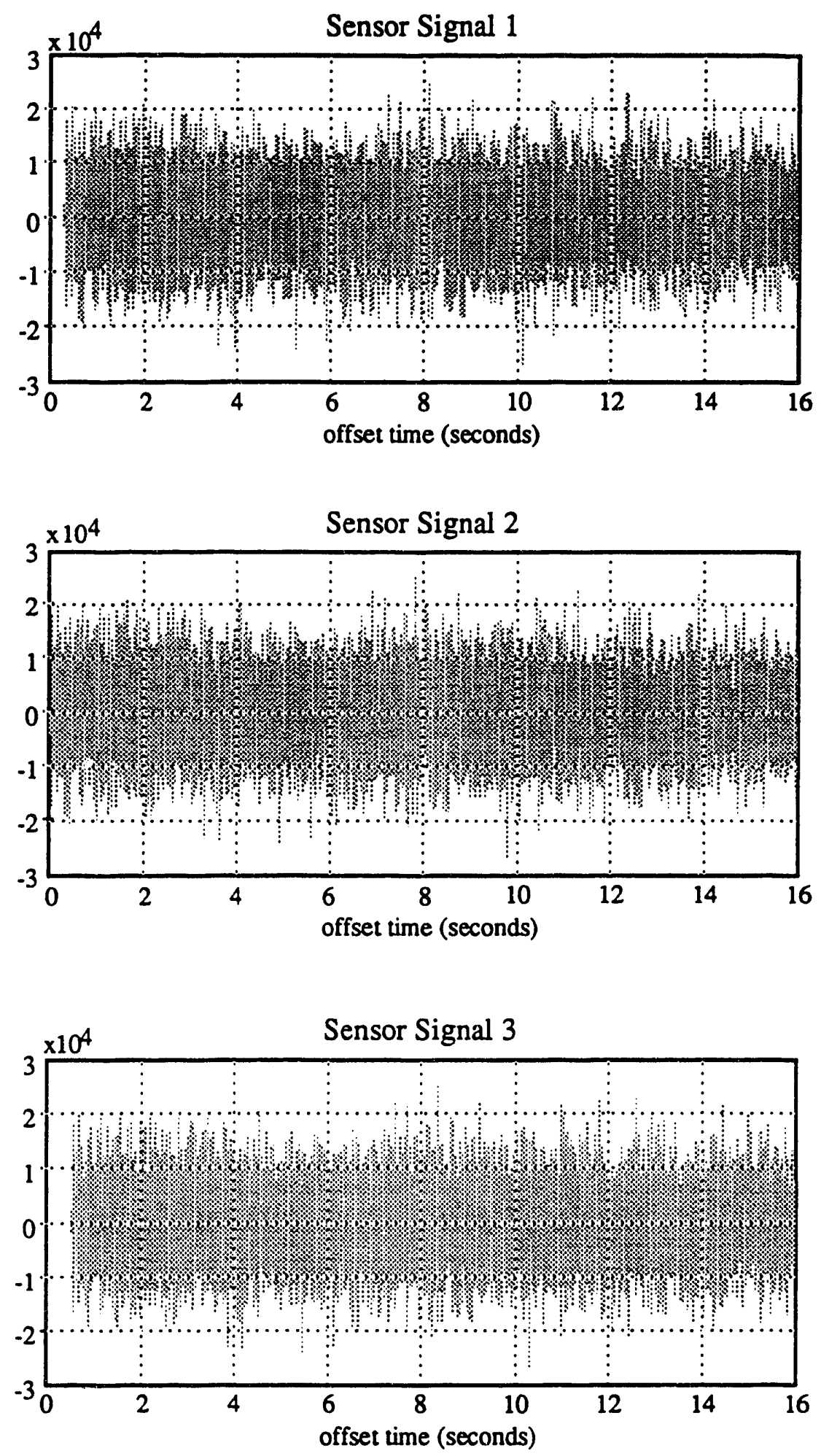

Fig. 5.2. Sensor signals for the example problem (con't) 

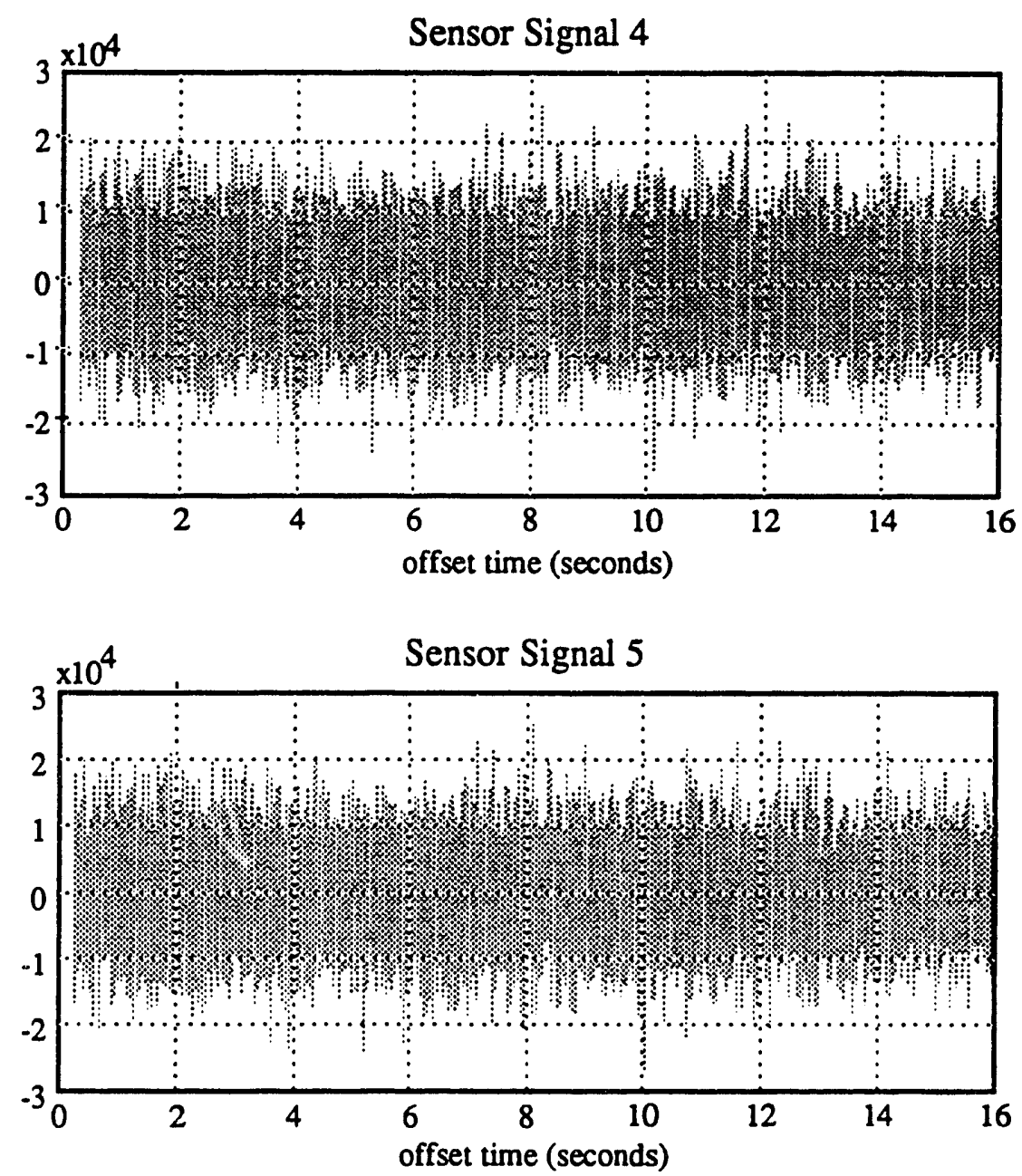

Fig. 5.2. Sensor signals for the example problem

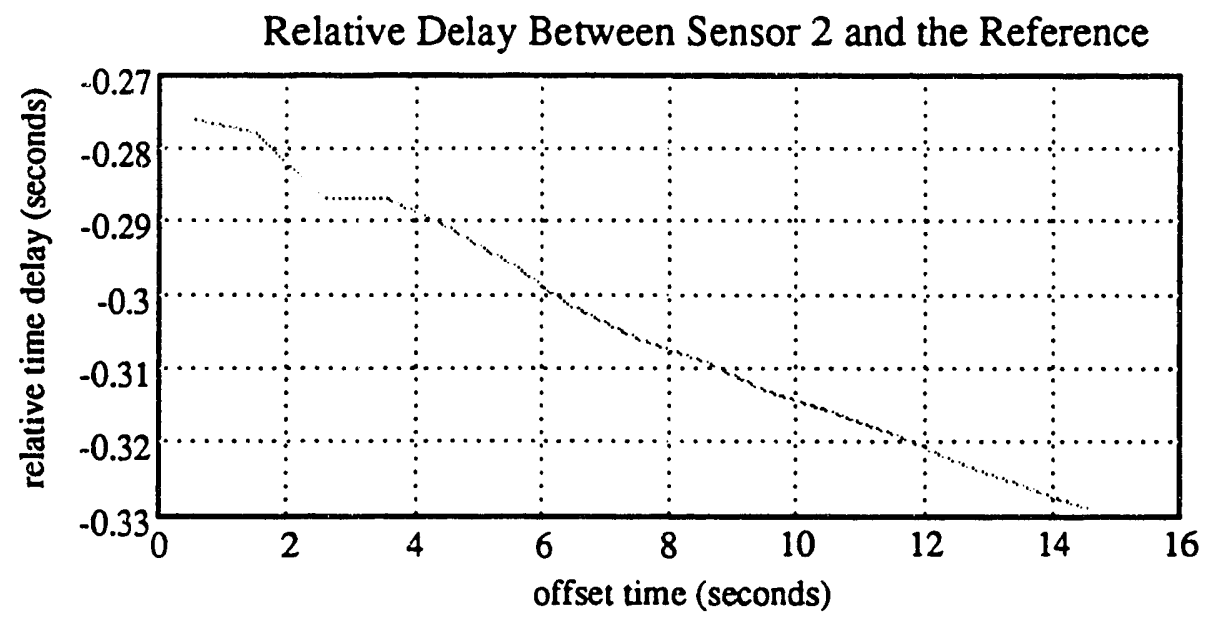

Fig. 5.3. Time delay between sensor signals and the reference sensor (sensor 1) for the example problem (con't) 

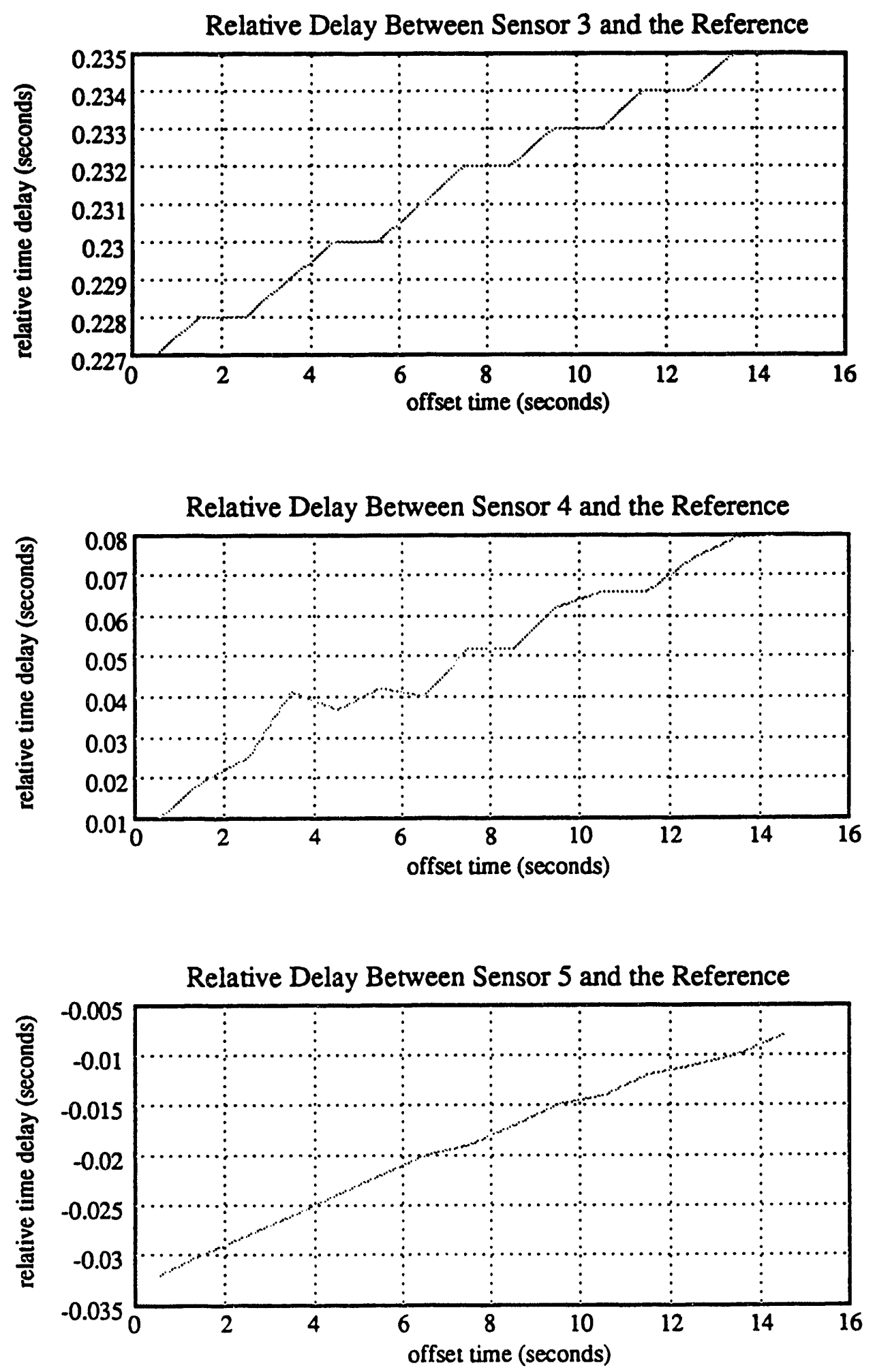

Fig. 5.3. Time delay between sensor signals and the reference sensor (sensor 1) for the example problem 

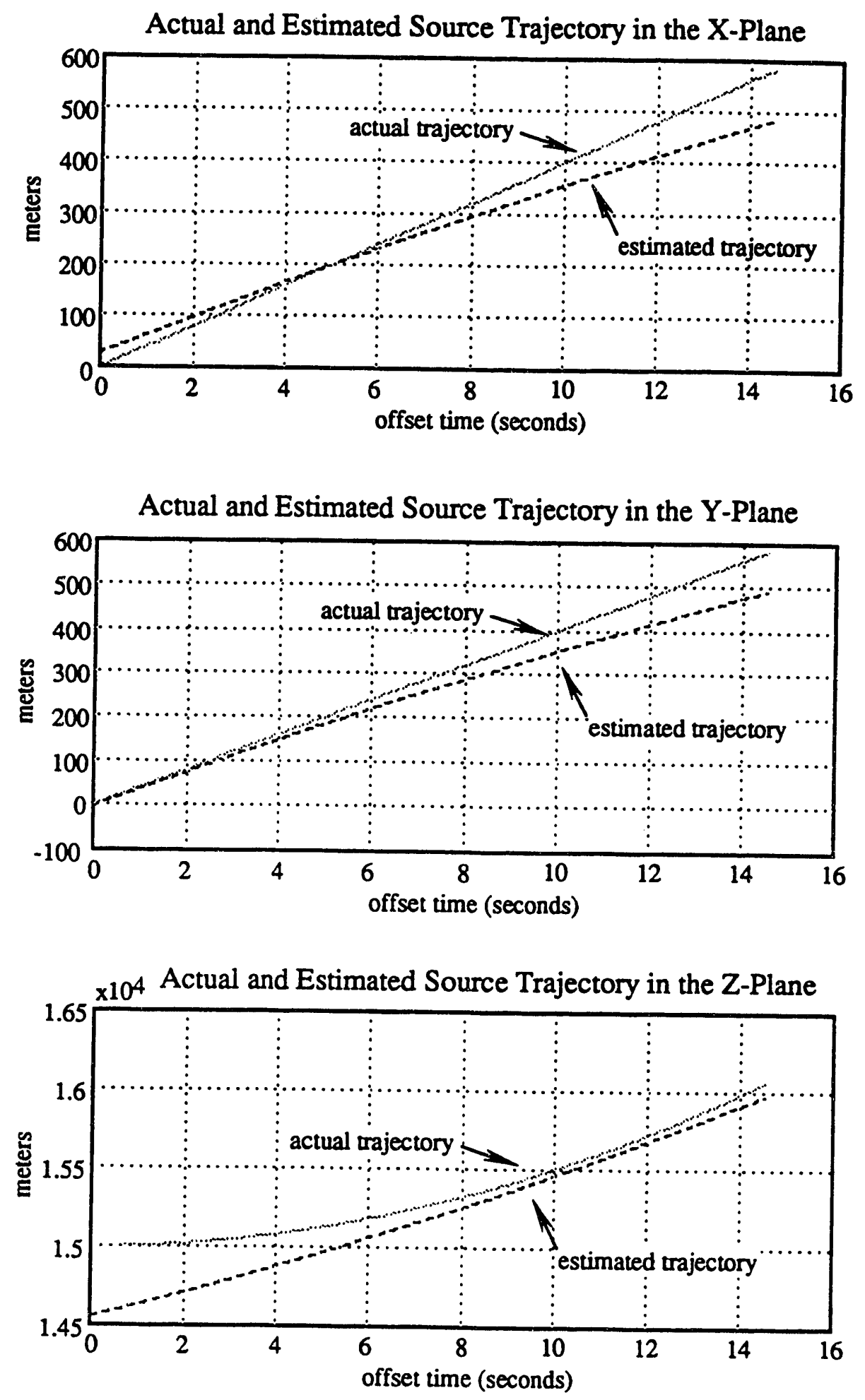

Fig. 5.4. Estimated and actual source trajectories for the example problem 
Second, the results may be saved into a file which can be used as input into additional signal processing algorithms such as Kalman Filters. The variables which are saved are given in Table 5.6.

\begin{tabular}{|c|l|}
\hline Variable Name & \multicolumn{1}{|c|}{ Description } \\
\hline Time_Comp & $\begin{array}{l}\text { The time (offset) at which the source locations are } \\
\text { estimated by the passive localization (seconds). }\end{array}$ \\
\hline X_Comp & $\begin{array}{l}\text { The X-plane locations of the source estimated by } \\
\text { the passive localization (meters). }\end{array}$ \\
\hline Y_Comp & $\begin{array}{l}\text { The Y-plane locations of the source estimated by } \\
\text { the passive localization (meters). }\end{array}$ \\
\hline Z_Comp & $\begin{array}{l}\text { The Z-plane locations of the source estimated by } \\
\text { the passive localization (meters). }\end{array}$ \\
\hline Rel_Delay & $\begin{array}{l}\text { The relative time delay between the sensor signals } \\
\text { and the reference sensor signals (seconds). }\end{array}$ \\
\hline Time & $\begin{array}{l}\text { The time (offset) at which the source locations are } \\
\text { estimated after post processing (seconds). }\end{array}$ \\
\hline Traj_X_Comp & $\begin{array}{l}\text { The X-plane locations of the source estimated after } \\
\text { post processing (meters). }\end{array}$ \\
\hline Traj_Y_Comp & $\begin{array}{l}\text { The Y-plane locations of the source estimated after } \\
\text { post processing (meters). }\end{array}$ \\
\hline Traj_Z_Comp & $\begin{array}{l}\text { The Z-plane locations of the source estimated after } \\
\text { post processing (meters). }\end{array}$ \\
\hline
\end{tabular}

Table. 5.6. Variable which are saved into result file

The variables "X_Comp", "Y_Comp", and "Z_Comp" are one-dimensional arrays which contain the estimated source locations which are computed by the passive localization algorithm. These may be referred to as the pre-processed source locations. By comparison, the post-processed source locations are stored in the one-dimensional arrays"Traj_X_Comp", "Traj_Y_Comp", and "Traj_Z_Comp". The times which correspond to the pre-processed source locations are given in "Time_Comp", while the 
times which correspond to the post-processed source locations are given in "Time". Finally, "Rel_Delay" is a two-dimensional array where the entry $(\mathrm{i}, \mathrm{j})$ represents the $\mathrm{i}^{\text {th }}$ relative time delay between sensor signal " $\mathrm{j}$ " and the reference signal. This $\mathrm{i}^{\text {th }}$ relative time delay occurs at the time given by the ith entry of "Time_Comp".

\subsection{Availability}

For more information regarding this program and its availability contact

Michael Buhl

Electronics Engineering

Engineering Research Division

Signal and Image Processing Research Group. 


\section{REFERENCES}

[1] J.V. Candy, Signal Processing - The Modern Approach. McGraw-Hill, 1988.

[2] G.M. Jenkins and D.G. Watts, Spectral Analysis and Its Applications. San Francisco, CA: Holden-Day, 1968.

[3] L.H. Koopmans, The Spectral Analysis of Time Series. New York: Academic, 1974.

[4] G.C. Carter and C.H. Knapp, "The generalized correlation method for estimation of time delays", IEEE Trans. Acoust., Speech, Signal Processing, vol. ASSP-24, pp. 320-327, August 1976.

[5] K. Scarbrough, N. Ahmed, G.C. Carter, "On the simulation of a class of time delay estimation algorithms", IEEE Trans. Acoust., Speech, Signal Processing, vol. ASSP-29, pp. 534-539, June 1981.

[6] P.R. Roth, "Effective measurements using digital signal analysis", IEEE Spectrum, vol. 8, pp. 62-70, April 1971.

[7] G.C. Carter, A.H. Nuttall, and P.G. Cable, "The smoothed coherence transform", Proc. IEEE (Lett.), vol. 61, pp. 1497-1498, October 1973.

[8] G.C. Carter, A.H. Nuttall, and P.G. Cable, "The smoothed coherence transform", Naval Underwater Systems Center, New London, CT, Tech. Memo TC-15972, August 8, 1972.

[9] E.J. Hannan and P.J. Thomson, "Estimating group delay", Biometrika, vol. 60, pp. 241-253, 1973.

[10] Benjamin Friedlander, "A Passive Localization Algorithm and Its Accuracy Analysis", IEEE Journal of Oceanic Engineering, vol. OE-12, pp.234-244, January 1987.

[11] Lawrence C. Ng and Yaakov Bar-Shalom, "Multisensor Multitarget Time Delay Vector Estimation", IEEE Trans. Acoust., Speech, Signal Processing, vol. ASSP34, pp. 669-678, August 1986. 
A. Menu Structure 


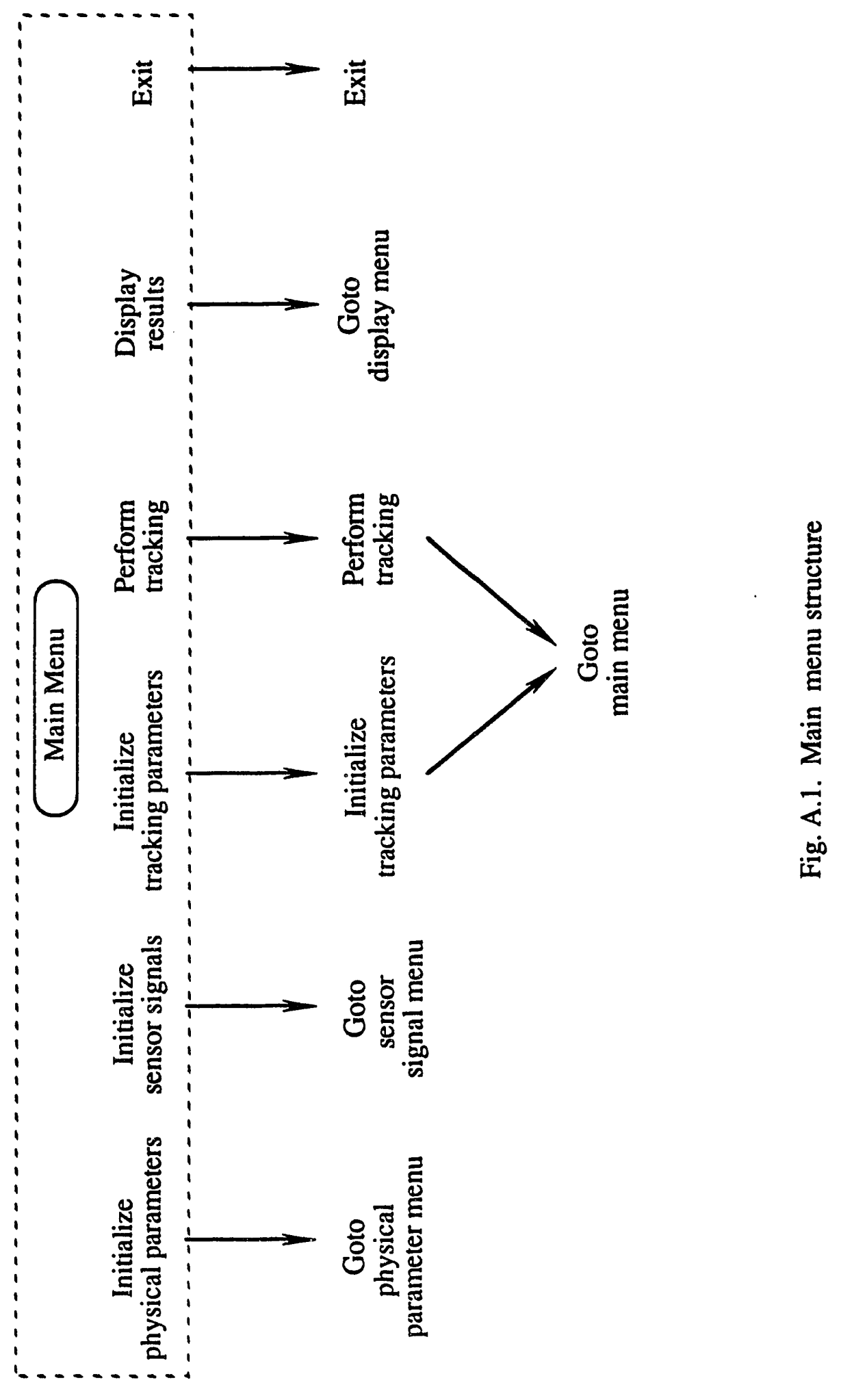





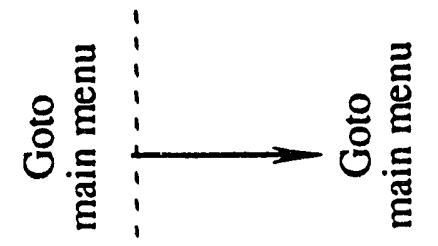

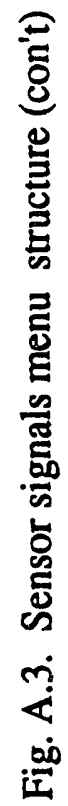




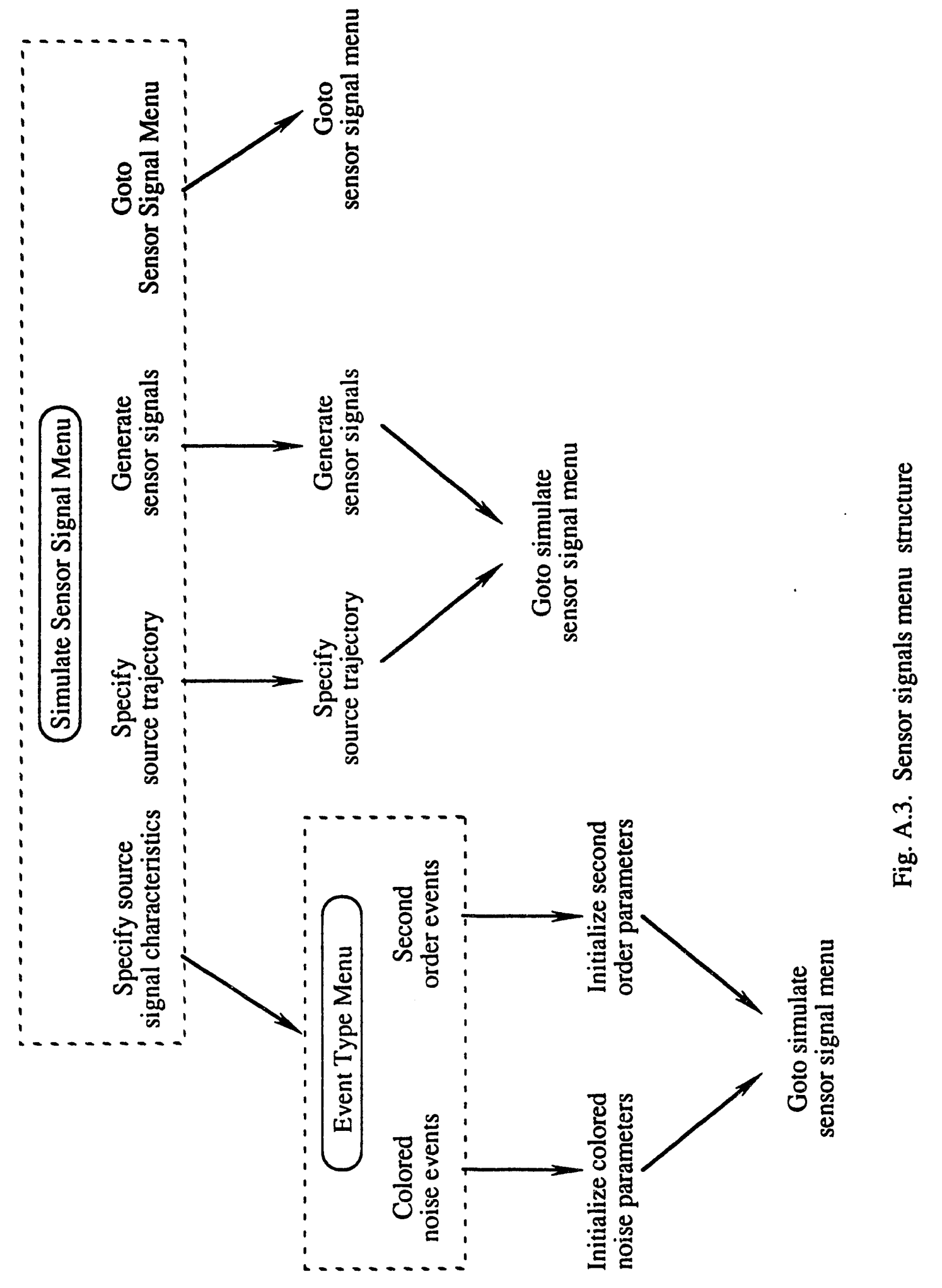



B. Source Code 


\section{B.1. track.m}

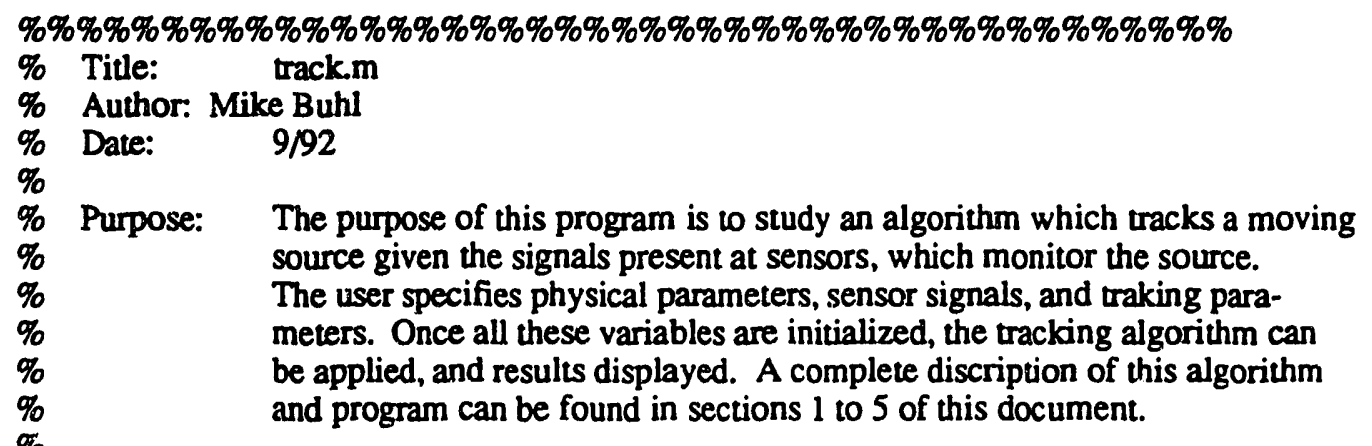

\%\%\%\%\%\%\%\%\%\%\%\%\%\%\%\%\%\%\%\%\%\%\%\%\%\%\%\%\%\%\%\%\%\%\%\%\%\%\%\%\%\%\%

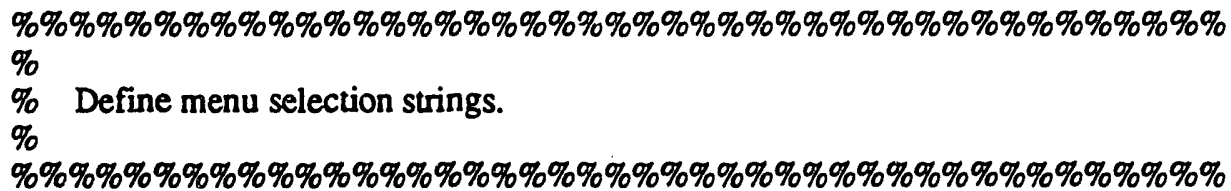

menu0_1 = 'Initialize physical parameters';

menu0_2 = 'Initialize sensor signals';

menu0_3 = Tnitialize tracking parameters';

menu0_4 = 'Perform tracking';

menu0_5 = 'Display results';

menu0_6 = 'Exit';

menul_1 = 'Load physical parameters';

menu1_2 = 'Specify physical parameters';

menul_3 = 'Goto main menu';

menu2_1 = 'Load sensor signals';

menu2_2 = 'Specify sensor signals';

menu2_3 = 'Goto main menu';

menu3_1 = 'Specify source signal characteristics';

menu3_2 = 'Specify source trajectory';

menu3_3 = 'Generate sensor signais';

menu3_4 = 'Goto sensor signal menu';

menu4_1 = 'Colored noise events';

menu4_2 = 'Second order events';

menus_1 = 'Fixed window length';

menus_2 = 'Adaptive window length';

menu6_1 = 'Unity gain';

menu6_2 = 'Roth impulse response';

menu6_3 = 'Smoothed coherence transform (SCOT)';

menu6_4 = 'Phase transform (PHAT)';

menu6_5 = 'Maximum likelihood (MI)';

menu7_1 = 'Sensor signals'; 
menu7_2 = 'Time delay estimates';

menu7_3 = 'Source trajectories';

menu7_4 = 'Goto main menu';

\%\%\%\%\%\%\%\%\%\%\%\%\%\%\%\%\%\%\%\%\%\%\%\%\%\%\%\%\%\%\%\%\%\%\%\%\%\%\%\%\%\%\%

$\%$

$\%$ Initialize flag variables.

$\%$

\%\%\%\%\%\%\%\%\%\%\%\%\%\%\%\%\%\%\%\%\%\%\%\%\%\%\%\%\%\%\%\%\%\%\%\%\%\%\%\%\%\%\%

Para_Spec =0;

Sig_Spec = 0;

Traj_Spec $=0$;

Track_Spec $=0$;

Event_Type $=0$;

Sen_Sig_Valid $=0$;

Track_Valid $=0$;

\%\%\%\%\%\%\%\%\%\%\%\%\%\%\%\%\%\%\%\%\%\%\%\%\%\%\%\%\%\%\%\%\%\%\%\%\%\%\%\%\%\%\% $\%$

\% Display the Main Menu.

\%\%\%\%\%\%\%\%\%\%\%\%\%\%\%\%\%\%\%\%\%\%\%\%\%\%\%\%\%\%\%\%\%\%\%\%\%\%\%\%\%\%\%

Select_0 $=0$;

while (Select_0 $=6$ )

clc;

Select_0 = menu('Main Menu',menu0_1,menu0_2,menu0_3,menu0_4,menu0_5, ... menu0_6);

\%\%\%\%\%\%\%\%\%\%\%\%\%\%\%\%\%\%\%\%\%\%\%\%\%\%\%\%\%\%\%\%\%\%\%\%\%\%\%\%\%\%\%

$\%$

\% Main Menu $=>$ Specify physical parameters

$\% \quad$ Display the Physical Parameters Menu.

$\%$

\%\%\%\%\%\%\%\%\%\%\%\%\%\%\%\%\%\%\%\%\%\%\%\%\%\%\%\%\%\%\%\%\%\%\%\%\%\%\%\%\%\%\%

if $($ Select_ $0=1)$

Select_1 $1=0$;

while $($ Select_I $=3)$

clc;

Select_1 = menu('Physical Parameters Menu',menul_1,menu1_2,menul_3);

\%\%\%\%\%\%\%\%\%\%\%\%\%\%\%\%\%\%\%\%\%\%\%\%\%\%\%\%\%\%\%\%\%\%\%\%\%\%\%\%\%\%\%

$\%$

\% Physical Parameters Menu $=>$ Load physical parameters

$\%$ Load the number of sensors, sensor coordinates, signal velocity, signal

\% attenuation constant, sampling period, and variance of sensor signal noise

$\%$ from a parameter file.

$\%$

\%\%\%\%\%\%\%\%\%\%\%\%\%\%\%\%\%\%\%\%\%\%\%\%\%\%\%\%\%\%\%\%\%\%\%\%\%\%\%\%\%\%\%

if $($ Select_1 $1=1)$

clc;

File_Name = input('Enter the parameter file name: ','s');

Ins_Arg = [File_Name '.mat']; 
Ins_Arg $=$ [File_Name '.mat Number_Sen Sen_Coordinates Signal_Vel

Signal_Att Sampling_Per Var_Sen_Noise'];

Save_Ins = ['save ' Ins_Arg];

end

eval(Save_Ins);

end

end

\%\%\%\%\%\%\%\%\%\%\%\%\%\%\%\%\%\%\%\%\%\%\%\%\%\%\%\%\%\%\%\%\%\%\%\%\%\%\%\%\%\%\%

$\%$

\% Main Menu $=>$ Specify sensor signals

$\%$ Display the Sensor Signal Menu.

$\%$

\%\%\%\%\%\%\%\%\%\%\%\%\%\%\%\%\%\%\%\%\%\%\%\%\%\%\%\%\%\%\%\%\%\%\%\%\%\%\%\%\%\%\%

elseif (Select_0 $=2$ )

if $($ Para_Spec $=1)$

Select_2 $=0$;

while (Select_2 $=3$ )

clc;

Select_2 = menu('Sensor Signal Menu',menu2_1,menu2_2,menu2_3);

\%\%\%\%\%\%\%\%\%\%\%\%\%\%\%\%\%\%\%\%\%\%\%\%\%\%\%\%\%\%\%\%\%\%\%\%\%\%\%\%\%\%\%

$\%$

\% Sensor Signal Menu $\Rightarrow$ Load sensor signals

$\% \quad$ Load sensor signals

$\%$

\%\%\%\%\%\%\%\%\%\%\%\%\%\%\%\%\%\%\%\%\%\%\%\%\%\%\%\%\%\%\%\%\%\%\%\%\%\%\%\%\%\%\%

if $($ Select_ $2=1)$

clc;

File_Name $=$ input('Enter the sensor signal file name: ','s');

Ins_Arg = [File_Name '.mat'];

Load_Ins = ['load ' Ins_Arg];

eval(Load_Ins);

[Num_Sen_Data,Num_Points] = size(Sensor_Sig);

if (Num_Sen_Data $=$ Number_Sen)

fprintf(The sensor signals have been loaded.');

Signal_Len $=$ Num_Points*Sampling_Per;

Sen_Sig_Valid $=1$;

Track_Valid $=0$;

else

Track_Spec $=0$;

clc;

specified as a physical parameter);

fprintf( $V$ The number of sensor corresponding to the data is different than that end

dummy= input('Hit any key to continue ...');

\%\%\%\%\%\%\%\%\%\%\%\%\%\%\%\%\%\%\%\%\%\%\%\%\%\%\%\%\%\%\%\%\%\%\%\%\%\%\%\%\%\%\%

$\%$

$\%$ Sensor Signal Menu $=>$ Specify sensor signals

$\%$ Display Simulate Sensor Signals Menu.

$\%$

\%\%\%\%\%\%\%\%\%\%\%\%\%\%\%\%\%\%\%\%\%\%\%\%\%\%\%\%\%\%\%\%\%\%\%\%\%\%\%\%\%\%\%

elseif (Select_2 $=2$ ) 


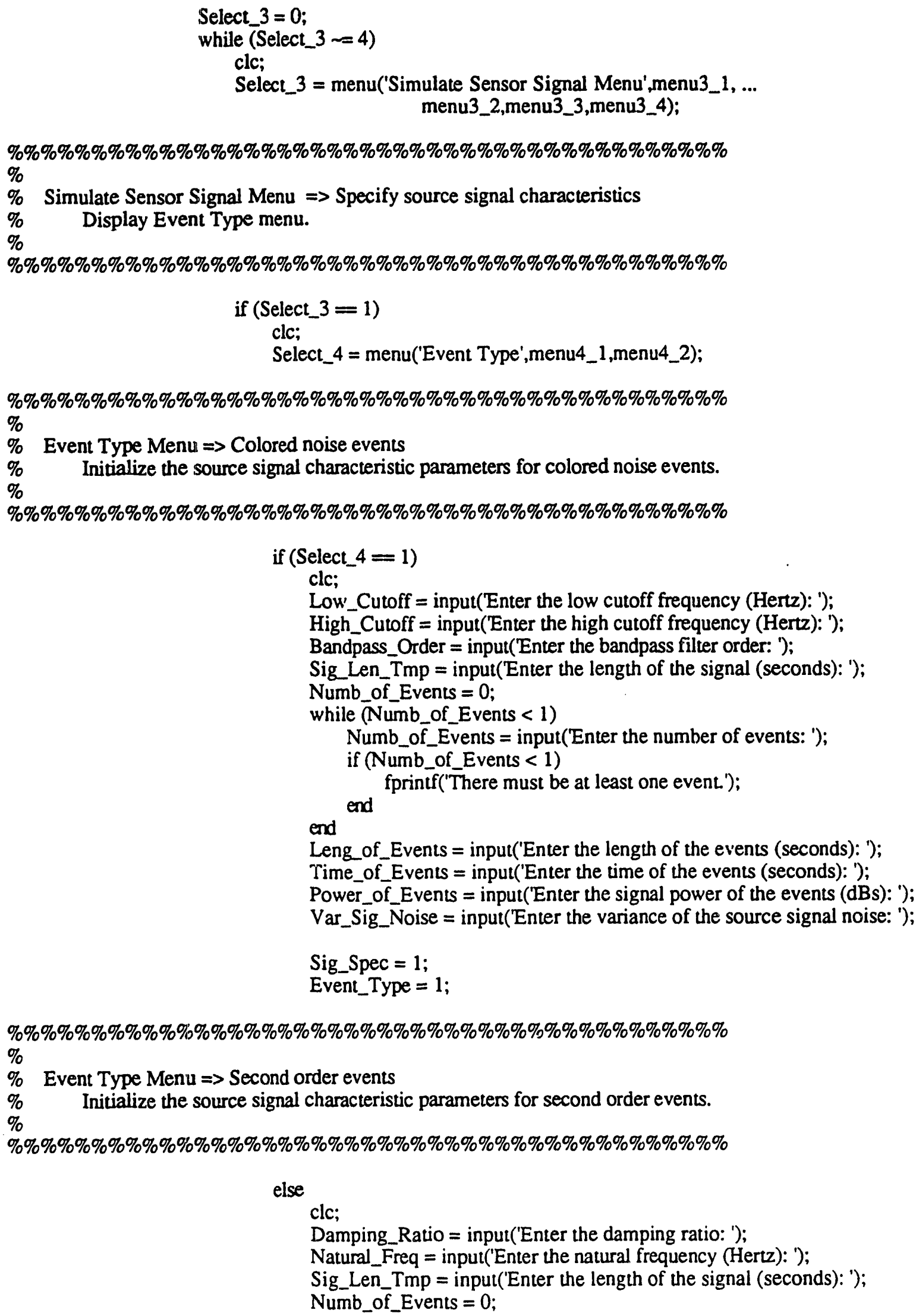


$[\mathrm{y} / \mathrm{n}] ?$ ?','s';

$$
\text { end }
$$

Damping_Ratio,Natural_Freq, ...

Time_of_Events, Power_of_Events, ...

Var_Sig_Noise);

Sensor_Sig $=$ sen_sig(Sen_Coordinates, ...

Signal_Vel,Sampling_Per,X_Coef, Y_Coef, ...

Z_Coef,Var_Sen_Noise,Signal_Att,Src_Sig);

Num_Points = length $($ Sensor_Sig $(1,:))$;

Data_Type $=2$;

Sen_Sig_Valid $=1$;

Track_Valid $=0$;

Track_Spec $=0$;

fprintf( ( $n$ The sensor signals have been generated. $n$ ');

answer $=$ ' $a$ ';

while ((answer $\sim$ 'y) \& (answer $\sim=$ ' $Y$ ') \& ...

(answer $\sim=$ ' $n$ ) \& (answer $\sim=$ N))

answer = input ('Do you want to save these sensor signals to a file

if ((answer $\left.==^{\prime} y^{\prime}\right) \mid\left(\right.$ answer $\left.\left.={ }^{\prime} Y\right)\right)$

File_Name = input('Enter the sensor signal file name: ','s'); Ins_Arg $=[$ File_Name '.mat Data_Type X_Coef Y_Coef

Z_Coef Sensor_Sig'];

Save_Ins = ['save ' Ins_Arg];

end eval(Save_Ins);

end

else

clc;

if $($ Sig_Spec $=0)$

fprintf('Source signal characteristics must be initialized first!n'); end

if (Traj_Spec $=0$ ) end fprintf(' InSource trajectory must be initialized first!'); end

dummy = input('Hit any key to continue ...');

end

end

end

end

else

clc;

end

fprintf( ' $n$ Physical parameters must be initialized first!');

\%\%\%\%\%\%\%\%\%\%\%\%\%\%\%\%\%\%\%\%\%\%\%\%\%\%\%\%\%\%\%\%\%\%\%\%\%\%\%\%\%\%\%

$\%$

$\%$ Main Menu $=>$ Specify tracking parameters

$\% \quad$ Initialize the tracking parameters.

$\%$

\%\%\%\%\%\%\%\%\%\%\%\%\%\%\%\%\%\%\%\%\%\%\%\%\%\%\%\%\%\%\%\%\%\%\%\%\%\%\%\%\%\%\%

elseif (Select_0 $==3$ )

if (Sen_Sig_Valid $=1)$

clc; 


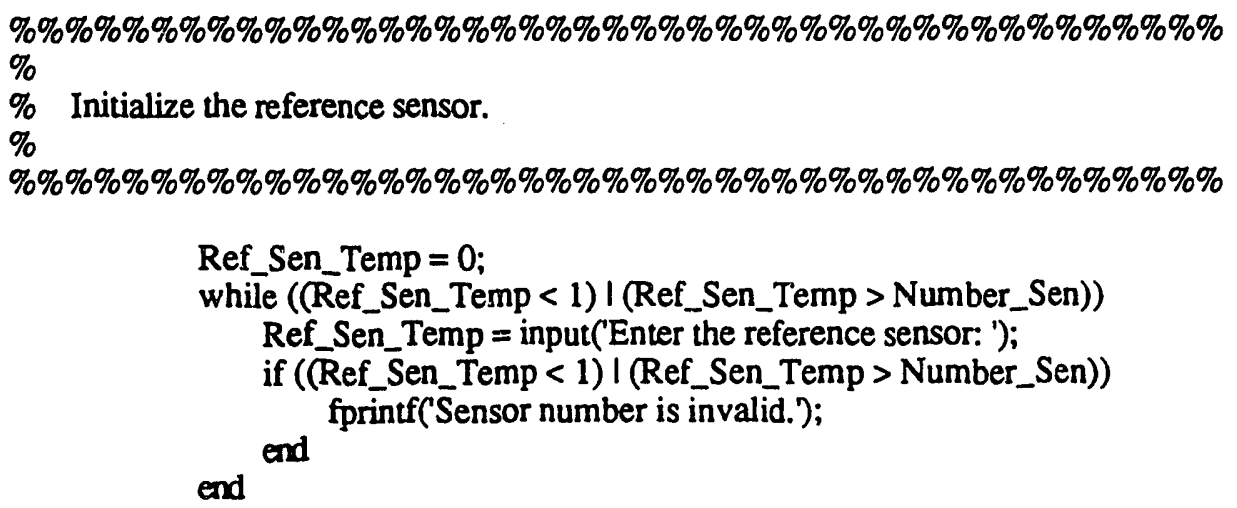

\%\%\%\%\%\%\%\%\%\%\%\%\%\%\%\%\%\%\%\%\%\%\%\%\%\%\%\%\%\%\%\%\%\%\%\%\%\%\%\%\%\%\%

$\%$

$\%$ Initialize the period of the computed trajectory points.

$\%$

\%\%\%\%\%\%\%\%\%\%\%\%\%\%\%\%\%\%\%\%\%\%\%\%\%\%\%\%\%\%\%\%\%\%\%\%\%\%\%\%\%\%\%

Comp_Per = input('Enter the period of trajectory point computations (seconds): ');

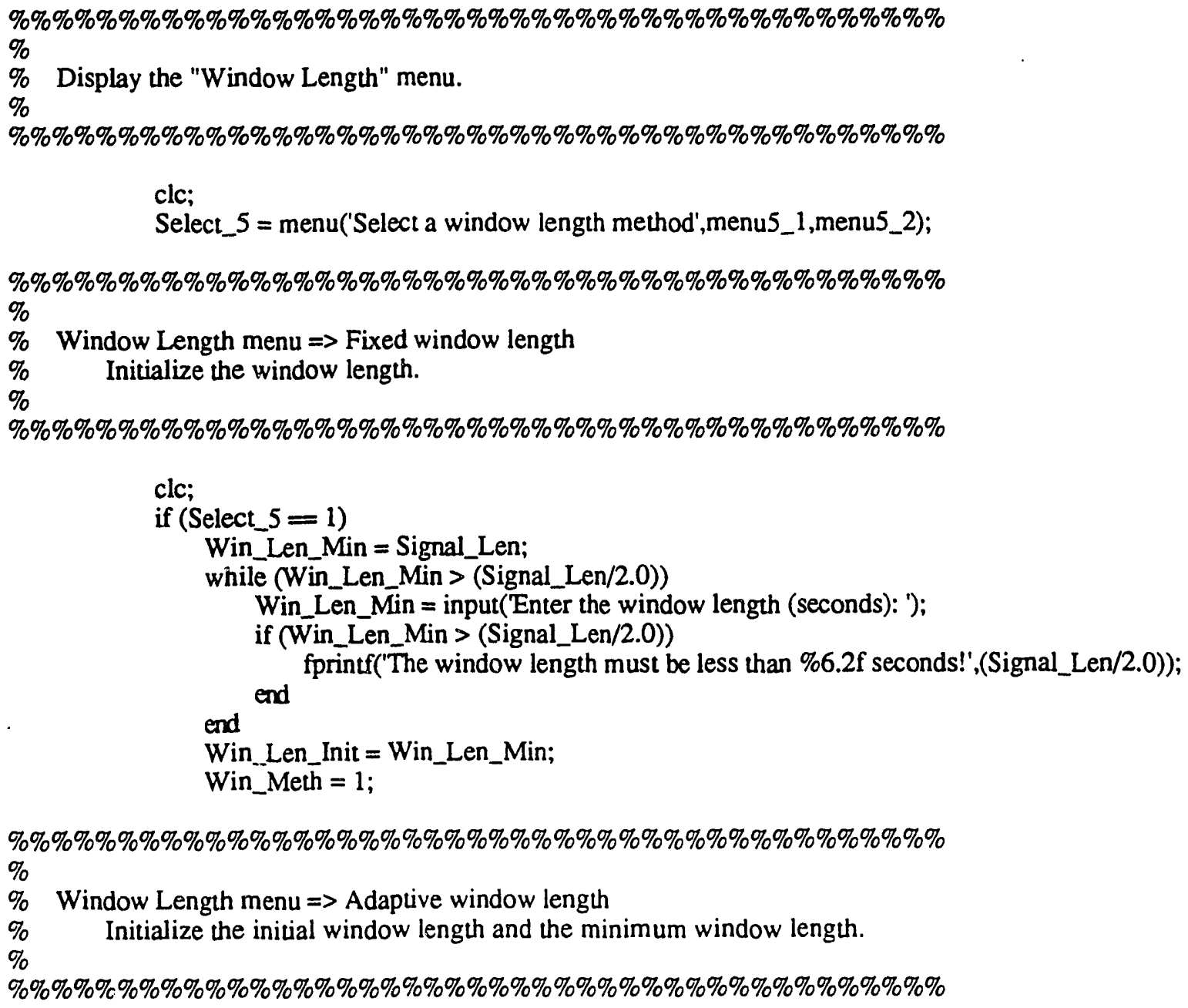


else

Win_Len_Init $=$ Signal_Len;

while (Win_Len_Init > (Signal_Len/2.0))

Win_Len_Init = input('Enter the initial window length (seconds): ');

if (Win_Len_Init > (Signal_Len/2.0)) end

fprintf('The window length must be less than \%6.2f seconds!',(Signal_Len/2.0));

end

Win_Len_Min = input('Enter the minimum window length (seconds): ');

end

Win_Meth $=2$;

\%\%\%\%\%\%\%\%\%\%\%\%\%\%\%\%\%\%\%\%\%\%\%\%\%\%\%\%\%\%\%\%\%\%\%\%\%\%\%\%\%\%\%

$\%$

\% Select a time delay processor.

$\%$

\%\%\%\%\%\%\%\%\%\%\%\%\%\%\%\%\%\%\%\%\%\%\%\%\%\%\%\%\%\%\%\%\%\%\%\%\%\%\%\%\%\%\%

clc;

Select_6 $=$ menu('Select a time delay processor',menu6_l,menu6_2, $\ldots$

Time_Est_Meth = Select_6; menu6_3,menu6_4,menu6_5);

Track_Spec $=1$;

Track_Valid $=0$;

else

clc;

fprintf('Sensor signals must be initialized first');

end

dummy = input('Hit any key to continue ...');

\%\%\%\%\%\%\%\%\%\%\%\%\%\%\%\%\%\%\%\%\%\%\%\%\%\%\%\%\%\%\%\%\%\%\%\%\%\%\%\%\%\%\%

$\%$

$\%$ Main Menu $=>$ Perform tracking

$\% \quad$ Apply the tracking algorithm.

$\%$

\%\%\%\%\%\%\%\%\%\%\%\%\%\%\%\%\%\%\%\%\%\%\%\%\%\%\%\%\%\%\%\%\%\%\%\%\%\%\%\%\%\%\%

elseif (Select_0 $==4)$

clc;

if $($ Track_Spec $=1)$

Win_Len_Init_P $=$ round(Win_Len_Init/Sampling_Per);

Win_Len_Min_P = round(Win_Len_Min/Sampling_Per);

Ref_Sen $=$ Ref_Sen_Temp;

Part $=0$;

Start $=1$

Stop $=$ Win_Len_Init_P;

fprintf('The estimated relative delay vectors in seconds: $\left.n^{\prime}\right)$;

while (Stop < Num_Points)

Part $=$ Part +1

$x 1$ = Sensor_Sig(Ref Sen,Start:Stop);

for Sen_Num $=1$ :Number_Sen

if (Sen_Num $\sim=$ Ref_Sen)

$\times 2=$ Sensor_Sig(Sen_Num,Start:Stop); 


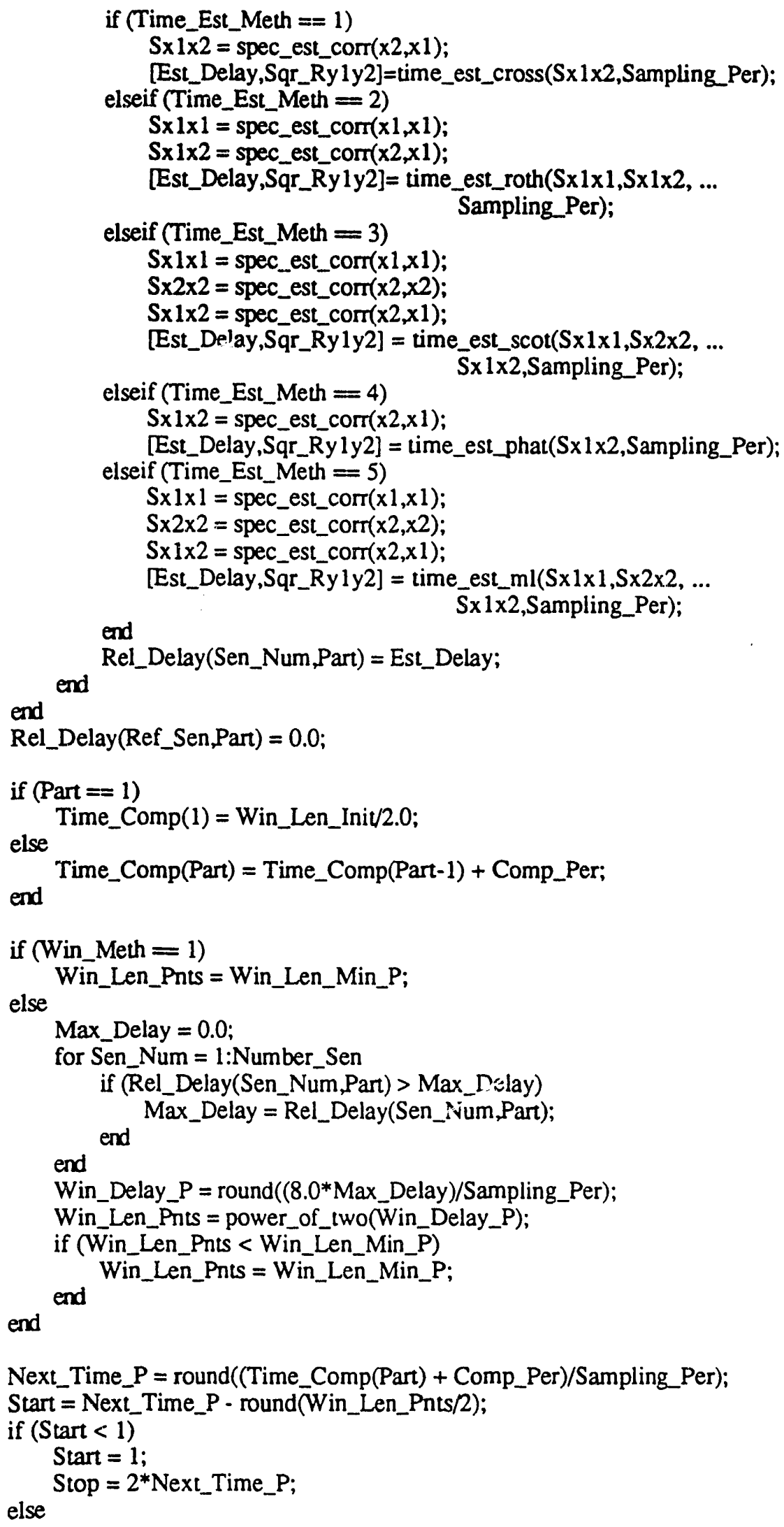

Next_Time_P $=$ round((Time_Comp (Part) + Comp_Per $) /$ Sampling_Per);

Start $=$ Next_Time_P - round $(W$ in_Len_Pnts $/ 2)$;

if $($ Start $<1)$

Start $=1$;

Stop $=2 *$ Next_Time_P;

else 

end

Stop $=$ Next_Time_P + round $($ Win_Len_Pnts/2) -1

fprintf( 'vAt time $=\% 6.2 \mathrm{f}$ seconds:',Time_Comp(Part));

for Sen_Num = 1:Number_Sen end fprintf(\%9.3F,Rel_Delay(Sen_Num,Part));

end

Num_Parts $=$ Part:

Rel_Delay $=$ Rel_Delay(:,1:Num_Parts);

Time_Comp = Time_Comp (1:Nurn_Parts);

clc;

fprintf( $v i n \backslash n$ The estimated source locations in meters: $\left.n^{\prime}\right)$;

for Part $=1:$ Num_Parts

Time_Delay = Rel_Delay(:,Part);

Est_Src_Cor $=$ locate_source(Sen_Coordinates,Ref_Sen,Time_Delay, ...

$X_{-}$Comp $($Part $)=$Est_Src_Cor(1); Signal_Vel);

$Y_{-}$Comp $(P a r t)=$ Est_Src_Cor $(2)$

Z_Comp (Part) $=$ Est_Src_Cor(3);

fprintf('VAt time $=\overline{\%} 6.2 \mathrm{f}$ seconds:', Time_Comp(Part));

fprintf('\%8.2f \%8.2f \%8.2f', X_Comp(Part), Y_Comp(Part), ...

end

Z_Comp(Part));

$X_{-}$Comp $=X_{-}$Comp (1:Num_Parts);

$Y_{-}$Comp $=Y_{-}$Comp $(1:$ Num_Parts);

Z_Comp $=$ Z_Comp(1:Num_Parts);

X_Coef_Comp $=$ polyfit $\left(\right.$ Time_Comp, $\left.X \_C o m p, 2\right)$;

Y_Coef_Comp $=$ polyfit (Time_Comp,Y_Comp,2);

Z_Coef_Comp = polyfit(Time_Comp,Z_Comp,2);

Num_Pnts_Trk $=$ round(Time_Comp(Num_Parts)/Sampling_Per);

$\mathrm{n}=0$ :Num_Pnts_Trk;

Time $=\mathrm{n} *$ Sampling_Per;

Traj_X_Comp = polyval(X_Coef_Comp,Time);

Traj_Y_Comp = polyval(Y_Coef_Comp,Time);

Traj_Z_Comp $=$ polyval(Z_Coef_Comp,Time);

if (Data_Type $=2$ )

Traj_X = polyval(X_Coef,Time);

Traj_ $Y=$ polyval $\left(Y_{-}\right.$Coef,Time);

end

Traj_Z = polyval $\left(Z_{-}\right.$Coef, Time);

Track_Valid $=1$;

clc;

fprintf( $v$ The tracking has been performed. $(n)$ );

answer $=$ ' $a$ ';

while ((answer $\sim=$ 'y') \& (answer $\sim=' Y$ ') \& ...

(answer $\sim=$ ' $n$ ') \& (answer $\sim=$ 'N'))

answer = input ('Do you want to save these results to a file $[y / n]$ ? ','s');

if $\left(\left(\right.\right.$ answer $\left.={ }^{\prime} y^{\prime}\right) \mid\left(\right.$ answer $\left.\left.=={ }^{\prime} Y^{\prime}\right)\right)$ 
File_Name $=$ input('Enter the result file name: ','s');

Ins_Arg $=$ [File_Name '.mat Time_Comp X_Comp Y_Comp Z_Comp Rel_Delay

Time Traj_X_Comp Traj_Y_Comp Traj_Z_Comp'];

end

Save_Ins = ['save ' Ins_Arg];

end eval(Save_Ins);

else

clc;

fprintf('Tracking parameters must be initialized first!');

end

dummy = input('Hit any key to continue ...');

\%\%\%\%\%\%\%\%\%\%\%\%\%\%\%\%\%\%\%\%\%\%\%\%\%\%\%\%\%\%\%\%\%\%\%\%\%\%\%\%\%\%\%

$\%$

Main Menu $\Rightarrow>$ Display Results

Display the Display Menu.

\%\%\%\%\%\%\%\%\%\%\%\%\%\%\%\%\%\%\%\%\%\%\%\%\%\%\%\%\%\%\%\%\%\%\%\%\%\%\%\%\%\%\%

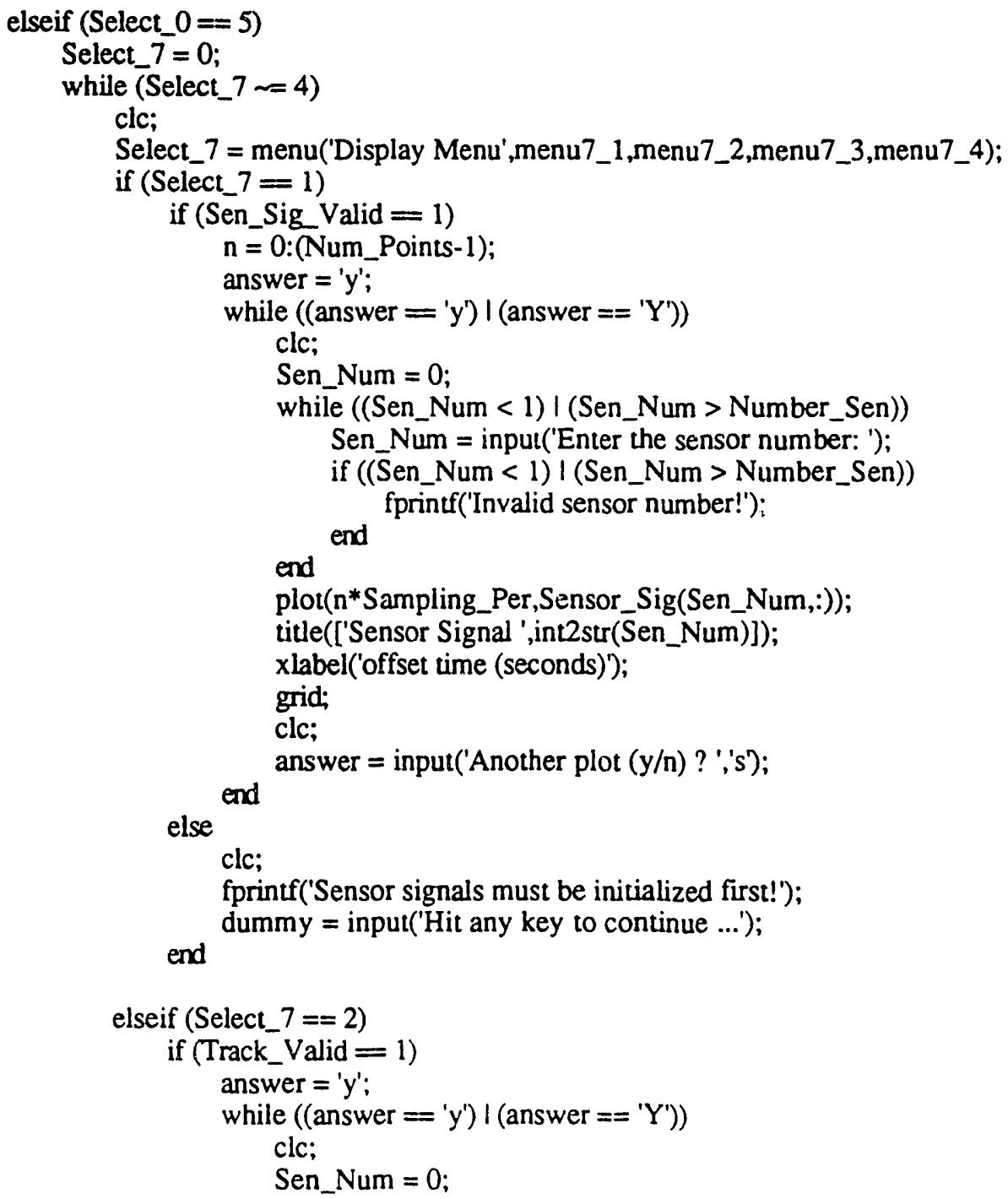


while ((Sen_Num $<1) \mid($ Sen_Num $>$ Number_Sen) $\mid($ Sen_Num $=$ Ref_Sen $))$

Sen_Num = input('Enter the sensor number (excluding refernece) : ');

if $(($ Sen_Num $<1) \mid($ Sen_Num $>$ Number_Sen $)$ ( $($ Sen_Num $==$ Ref_Sen $))$ end

fprintf('Invalid sensor number!');

end

plot(Time_Comp,Rel_Delay(Sen_Num,:));

title(['Relative Delay Between Sensor ',inDstr(Sen_Num),' and the Reference']);

xlabel('offset time (seconds));

ylabel('relative time delay (seconds));

grid;

clc;

end

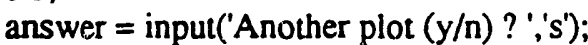

else

clc;

fprintf('The tracking algorithm has not been applied!');

end

dummy = input('Hit any key to continue ...');

elseif (Select_7 $==3$ )

if (Track_Valid $=1$ )

$\mathrm{n}=0$ :Num_Pnts_Trk;

if (Data_Type $=1$ )

plot $\left(n^{*}\right.$ Sampling_Per,Traj_X_Comp,' $g$ ');

title('Estimated Source Trajectory in the X-Plane');

xlabel('offset time (seconds)');

ylabel('meters');

grid;

clc;

dummy = input('Hit any key to continue ...');

plot(n*Sampling_Per,Traj_Y_Comp,' $g$ ');

title('Estimated Source Trajectory in the Y-Plane');

xlabel('offset time (seconds));

ylabel('meters');

grid:

clc;

dummy = input('Hit any key to continue ...');

plot(n*Sampling_Per,Traj_Z_Comp,'g');

title('Estir: ted Source Trajectory in the Z-Plane');

xlabel('offset time (seconds)');

ylabel('meters');

else

grid:

$\operatorname{plot}\left(n^{*}\right.$ Sampling_Per,Traj_X,'r', $n^{*}$ Sampling_Per, ...

Traj_X_Comp,'g');

title('Actual and Estimated Source Trajectory in the X-Plane');

xlabel('offset time (seconds)');

ylabel('meters');

grid;

clc;

dummy = input('Hit any key to continue ...');

plot(n*Sampling_Per,Traj_Y,' $r$ ', $n *$ Sampling_Per, ...

Traj_Y_Comp,' 'g');

title('Actual and Estimated Source Trajectory in the Y-Plane');

xlabel('offset time (seconds)');

ylabel('meters');

grid: 
clc; dummy = input('Hit any key to continue ...');

plot( $\mathrm{n}$ *Sampling_Per,Traj_Z,' $r$ ', $\mathrm{n}$ *Sampling_Per, ...

Traj_Z_Comp,' 'g');

title('Actual and Estimated Source Trajectory in the Z-Plane');

xlabel('offset time (seconds)');

end

ylabel('meters');

grid:

else

clc;

end

(racking algorithm has not been applied!')

end

dummy = input('Hit any key to continue ...');

end

end

end

B.2. src_sig_color.m

function Src_Sig $=$ src_sig_color(Signal_Len,Sampling_Per, $\ldots$

Leng_of_Events,_Low_Cutoff,High_Cutoff, ...

Bandpass_Order,Time_of_Events, ...

Power_of_Events,Var_Sig_Noise);

\%\%\%\%\%\%\%\%\%\%\%\%\%\%\%\%\%\%\%\%\%\%\%\%\%\%\%\%\%\%\%\%\%\%\%\%\%\%\%\%\%\%\%

$\%$

Title: src_sig_color

Author: Mike Buhl

Date: $\quad 9 / 92$

Purpose: This function generates the source signal. This signal consists of white noise events. The time at which these events occur and the signal power of these events are passed as arguments. Once the signal is constructed, unit variance white noise is added to it.

Inputs: Signal_Len = length of source signal (seconds)

Sampling_Per $=$ sample period (seconds)

Leng_of_Events = length of each event (seconds)

Low_Cutoff = low cutoff frequency for bandpass filter $(\mathrm{Hz})$

High_Cutoff = high cutoff frequency for bandpass filter $(\mathrm{Hz})$

Bandpass_Order $=$ bandpass filter order

Time_of_Events = time of events in $x 1$

Power_of_Events $=$ power of events in $\mathrm{dBs}$

Var_Sig_Noise $=$ variance of noise added to signal

Outputs: Src_Sig = source signal

\%\%\%\%\%\%\%\%\%\%\%\%\%\%\%\%\%\%\%\%\%\%\%\%\%\%\%\%\%\%\%\%\%\%\%\%\%\%\%\%\%\%\%

Event_Pnts $=$ round(Leng_of_Events/Sampling_Per);

Num_Pnts $=$ round(Signal_Len/Sampling_Per);

Loc1_Pnts = round(Time_of_Events/Sampling_Per);

Loc1_Pnts = Loc1_Pnis + 1; 
fcutoffs $=$ [2*Low_Cutof**Sampling_Per, 2*High_Cutoff*Sampling_Per];

$[b, a]=$ butter(Bandpass_Order,fcutoffs);

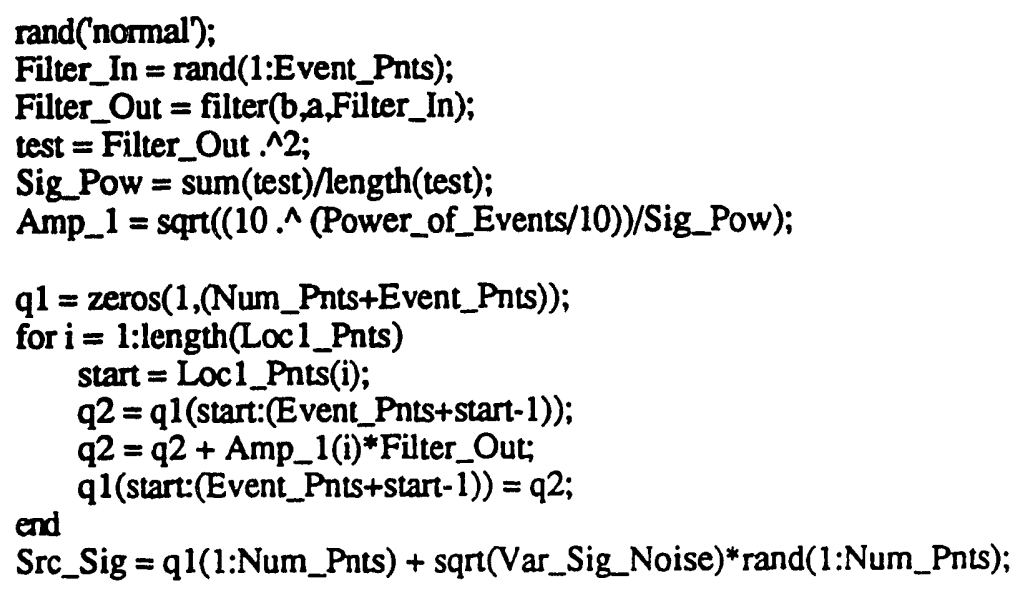

Src_Sig $=q 1\left(1: N u m \_P n t s\right)+$ sqrit(Var_Sig_Noise $)^{*}$ rand $(1:$ Num_Pnts $)$;

\section{B.3. src_sig_2order.m}

function Src_Sig $=$ src_sig_2order(Signal_Len,Sampling_Per,Damping_Ratio, ... Natural_Freq,Time_of_Events, ... Power_of_Events, Var_Sig_Noise);

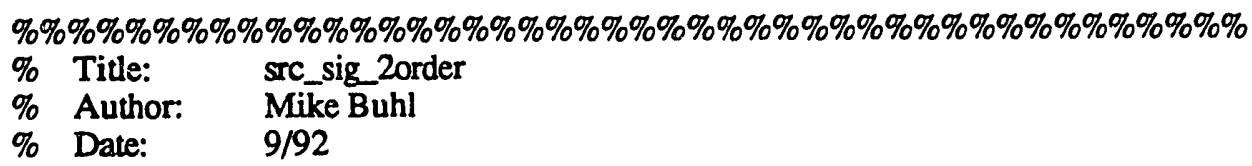

Num_Pnts $=$ round (Signal_Len/Sampling_Per);

Loc1_Pnts $=$ round(Time_of_Events/Sampling_Per);

Loc1_Pnts = Loc1_Pnts + 1;

WnT $=2 * \mathrm{pi}^{*}$ Natural_Freq $*$ Sampling_Per;

$a(1)=1$;

if Damping_Ratio $>1$

temp $=$ WnT*sqra (Damping_Ratio 2$)-1)$; $a^{\prime}(2)=-$ cxp (-Damping_Ratio* $\left.W n T\right) *(\exp (t e m p)+\exp (-t e m p))$; 
else

temp $=W n T^{*} \operatorname{sqr}(1$ - Damping_Ratio^2);

end

$a(2)=-2^{*} \exp (-$ Damping_Ratio* WnT $) * \cos (t e m p)$;

$a(3)=\exp (-2 *$ Damping_Ratio*WnT $)$;

$b(1)=1$;

Filter_In $=[1$ zeros(1:(Num_Pnts-1))];

test $=$ filter $(b, a, F i l t e r$ In $)$;

test $=$ test.$\wedge 2$;

Sig_Pow $=$ sum(test);

Amp_1 $1=\operatorname{sqrt}((10 . \wedge($ Power_of_Events/10))/Sig_Pow $)$;

Filter_In = zeros(1,Num_Pnts);

for $\mathrm{i}=1$ :length(Loc1_Pnts)

end

Filter_In(Loc1_Pnts(i)) = Amp_1(i);

Src_Sig = filter(b,a,Filter_In);

rand('normal');

Src_Sig = Src_Sig + sqrt (Var_Sig_Noise) ${ }^{*}$ rand $(1:$ Num_Pnts $)$;

B.4. sen_sig.m

function Sensor_Sig $=$ sen_sig(Sen_Coordinates,Signal_Vel,Sampling_Per,..

X_Coef,Y_Coef,Z_Coef,Var_Sen_Noise, ...

Signal_Att,Src_Sig)

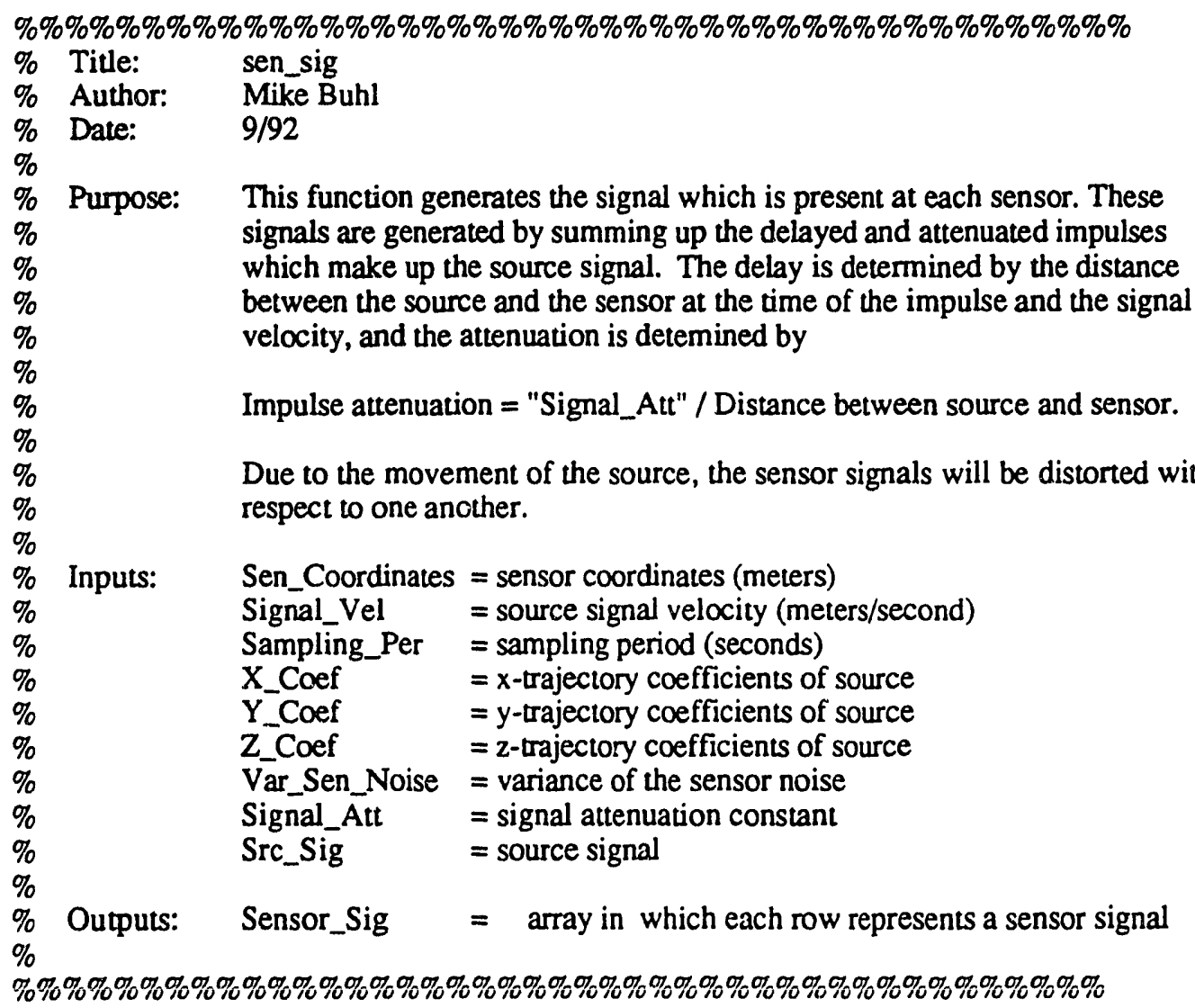




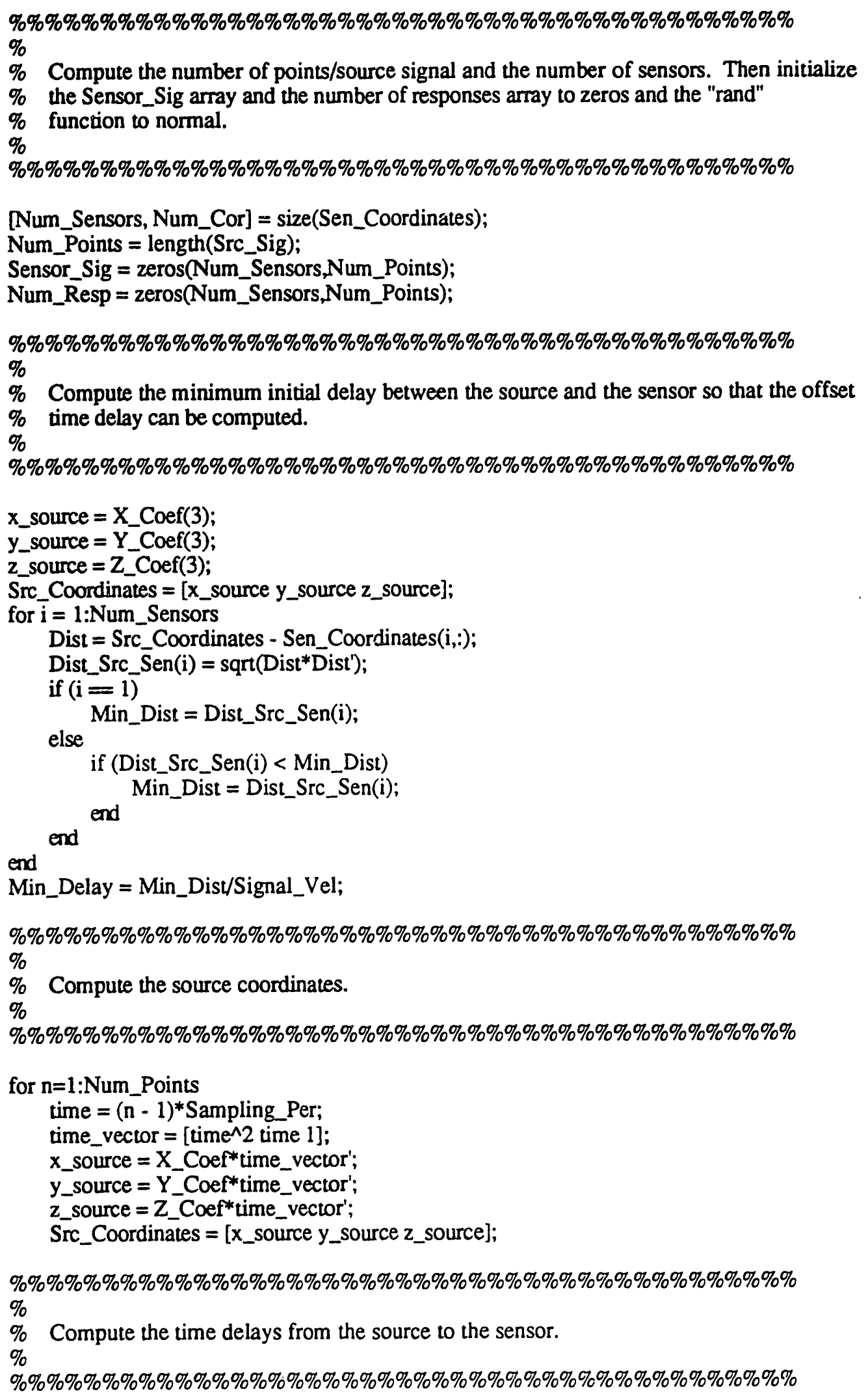




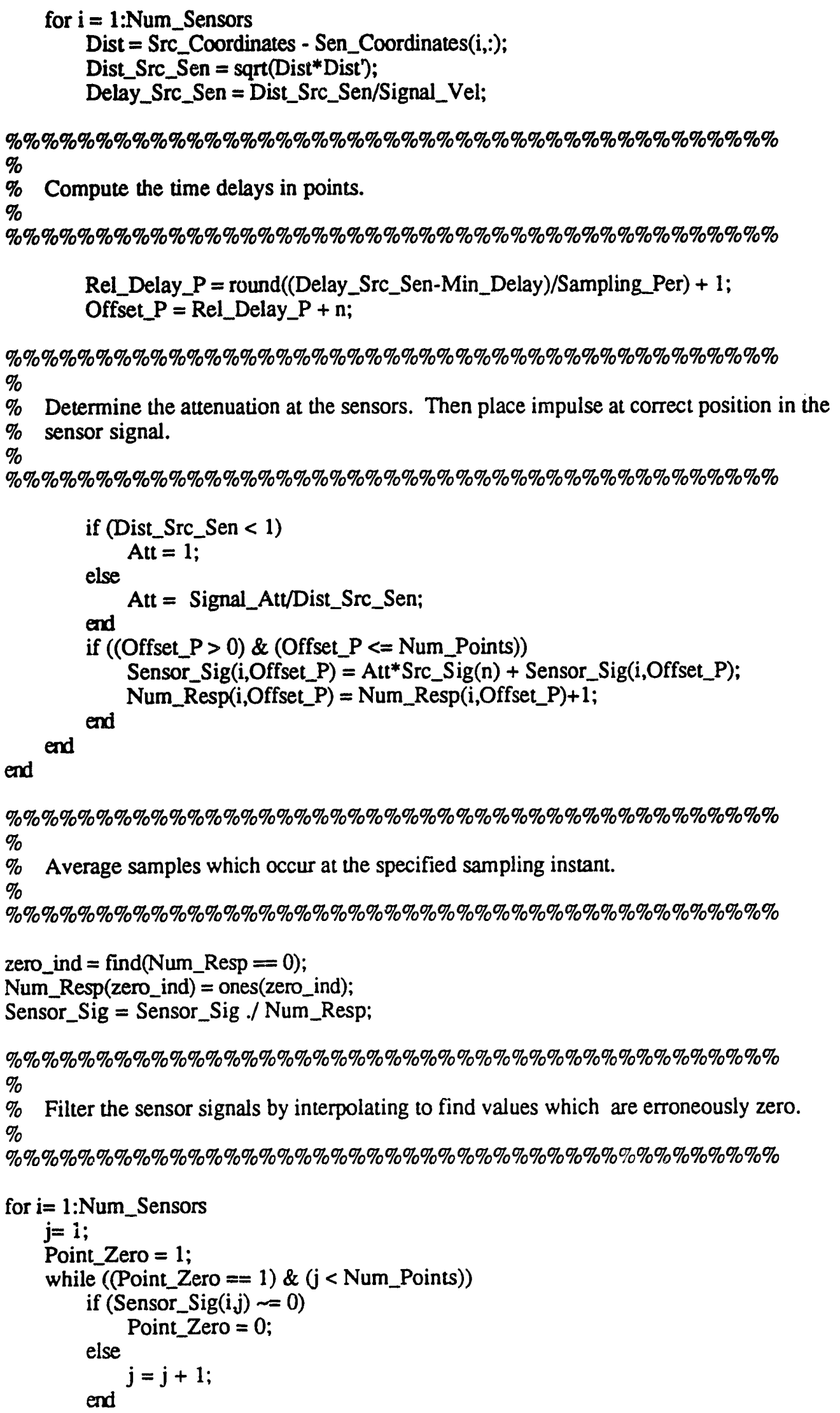


end

zero_ind $=$ find (Sensor_Sig(i,j:Num_Points $)=0$ );

for $\mathrm{k}=1$ :length(zero_ind)

index $=$ zero_ind $(k)+j-1$;

right_ind $=$ index;

while (Sensor_Sig(i,right_ind) $==0$ \& right_ind $<$ Num_Points)

end right_ind = right_ind +1 ;

slope $=($ Sensor_Sig(i,right_ind $)-$ Sensor_Sig(i,(index-1)) $) / . .$.

(right ind - index + 1);

end

end

Sensor_Sig $(\mathrm{i}$, index $)=$ slope + Sensor_Sig(i,(index-1));

rand('normal');

Sensor_Sig $=$ Sensor_Sig + sqrt(Var_Sen_Noise $)^{*}$ rand(Num_Sensors,Num_Points);

\section{B.5. spec_est_corr.m}

function $S \times 1 \times 2=$ spec_est_corr $(x 1, x 2)$

\%\%\%\%\%\%\%\%\%\%\%\%\%\%\%\%\%\%\%\%\%\%\%\%\%\%\%\%\%\%\%\%\%\%\%\%\%\%\%\%\%\%\%

$\%$

\% Title: spec_est_corr

Author: Mike Buhl

Date: $\quad 9 / 92$

Purpose: This function generates the power spectum and cross spectrum of $x 1$ and

$\mathrm{x} 2$. This is done using the Blackman-Tukey method. The correlation is

$\% \quad$ windowed with a hamming window.

$\%$ Inputs: $\quad x 1=$ array containing points of $x 1$

$\% \quad \mathrm{x} 2=$ array containing points of $\mathrm{x} 2$

$\%$ Outputs: $\quad \mathrm{S} \times 1 \times 2=$ cross spectrum between $\times 1$ and $\times 2$

$\%$

$\%$

\%\%\%\%\%\%\%\%\%\%\%\%\%\%\%\%\%\%\%\%\%\%\%\%\%\%\%\%\%\%\%\%\%\%\%\%\%\%\%\%\%\%\%

$\mathrm{N}=$ length $(\mathrm{x} 1)$;

$\mathrm{X} 1=\mathrm{fft}(\mathrm{x} 1,2 * \mathrm{~N})$

$\mathrm{X} 2=\mathrm{ftt}(\mathrm{x} 2,2 * \mathrm{~N})$

$\operatorname{Px} 1 \times 2=\mathrm{X} 1{ }^{*} \operatorname{conj}(\mathrm{X} 2)$;

$\operatorname{Rx} 1 \times 2=$ ifft(Pxilx2);

$R \times 1 \times 2=$ fftshift $(R \times 1 \times 2)$

$\operatorname{Rx} 1 \times 2=R \times 1 \times 2 *^{*}(\text { hamming }(2 * N))^{\prime}$

$\operatorname{Sx} 1 \times 2=\mathrm{fft}(\mathrm{R} \times 1 \times 2)$;

\section{B.6. time_est_cross.m}

function [Est_Delay, Sqr_Ry1 y2] = time_est_cross(Sx1x2,Sampling_For);

\%\%\%\%\%\%\%\%\%\%\%\%\%\%\%\%\%\%\%\%\%\%\%\%\%\%\%\%\%\%\%\%\%\%\%\%\%\%\%\%\%\%\% 


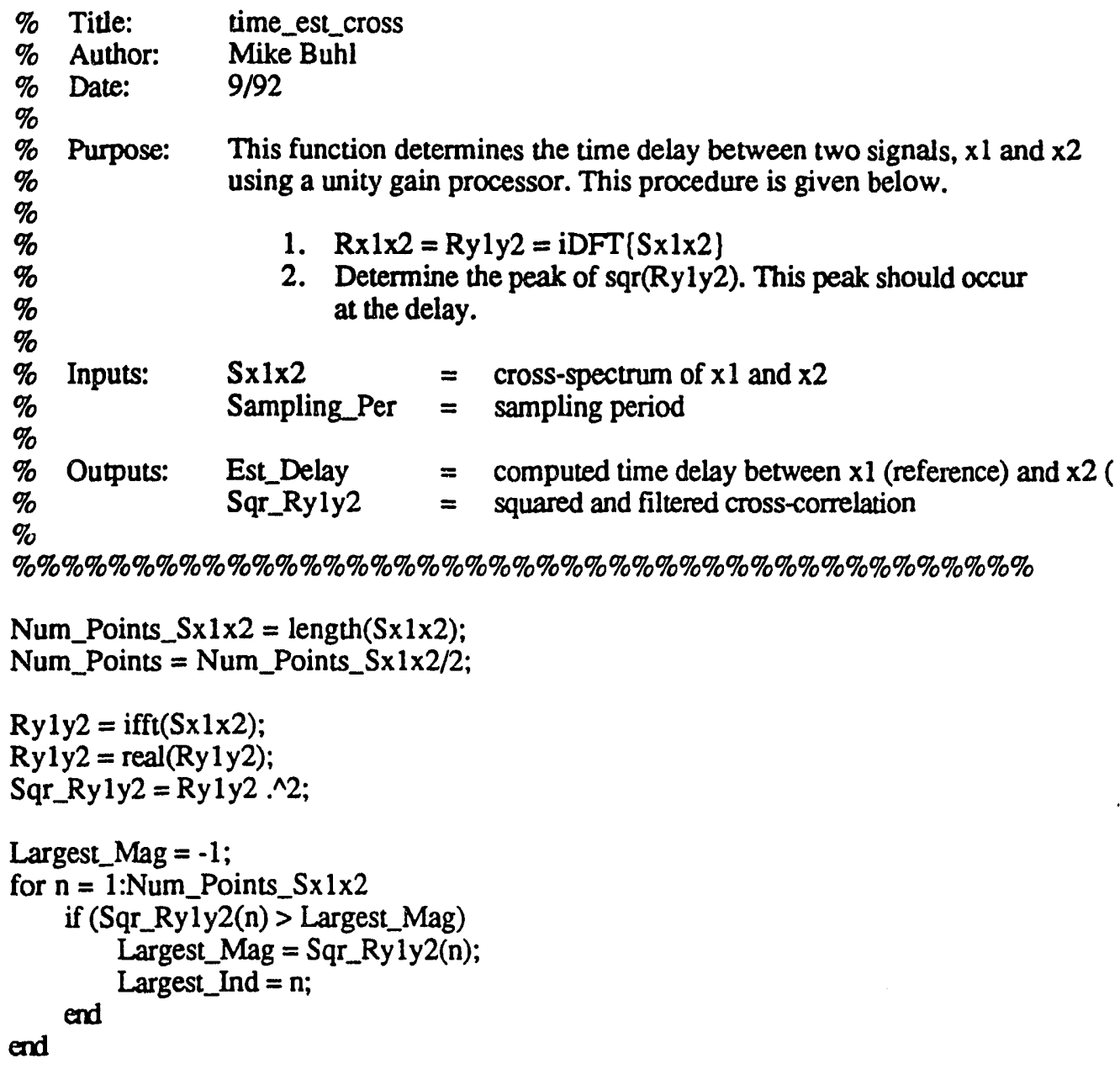

Est_Delay $=(($ Largest_Ind-1)-Num_Points $) *$ Sampling_Per,

\section{B.7. time_est_roth.m}

function [Est_Delay, Sqr_Ryly2]= time_est_roth(Sx1 x1,Sx1×2,Sampling_Per);

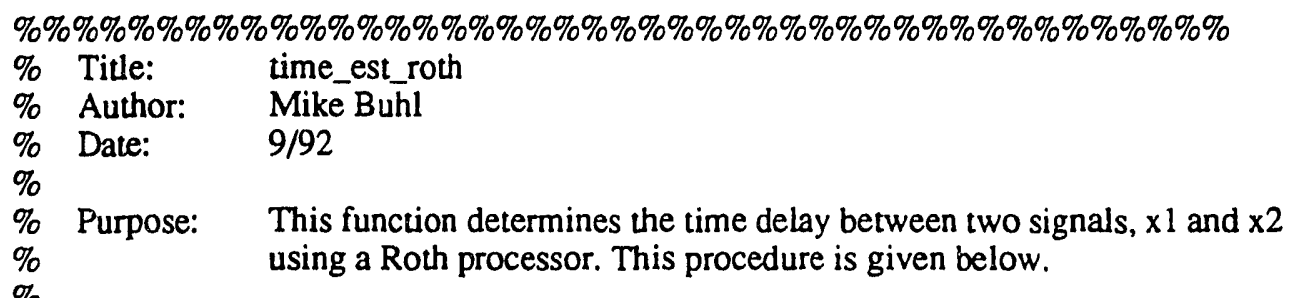

1. $S y 1 y 2 \approx S \times 1 \times 2 / S \times 1 \times 1$

2. Ryly2 = iDFT $\{$ Sy 1 y 2$\}$

3. Determine the peak of sqr(Ryly2). This peak should occur at the delay.
Inputs: $\quad S \times 1 \times 1=$ power-spectrum of $\times 1$
$\mathrm{S} \times 1 \times 2=$ cross-spectrum of $\times 1$ and $\times 2$
Sampling_Per $=$ sampling period




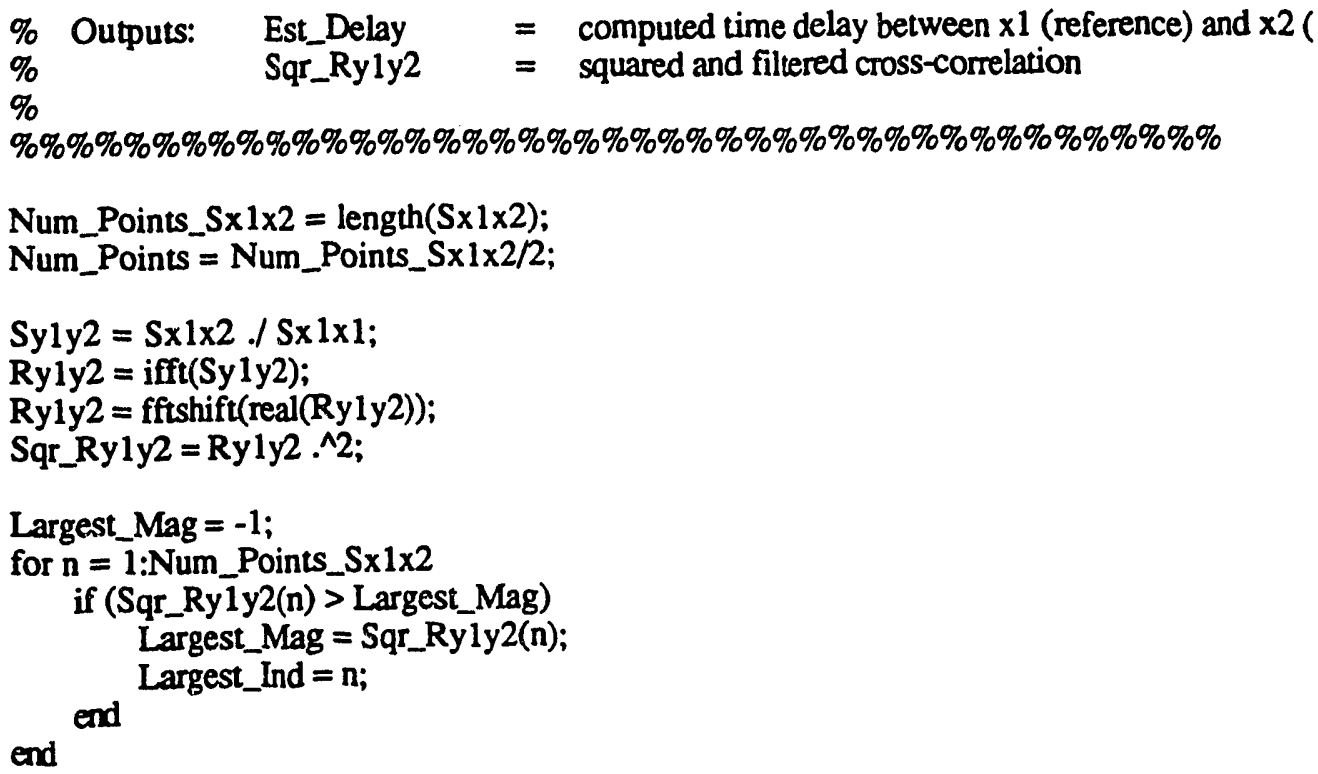

Est_Delay $=(($ Largest_Ind-1)-Num_Points $) *$ Sampling_Per,

\section{B.8. time_est_scot.m}

function [Est_Delay, Sqr_Ry1y2] $=$ time_est_scot $\left(S \times 1 \times 1, S \times 2 \times 2, S \times 1 \times 2, S a m p l i n g \_P e r\right)$;

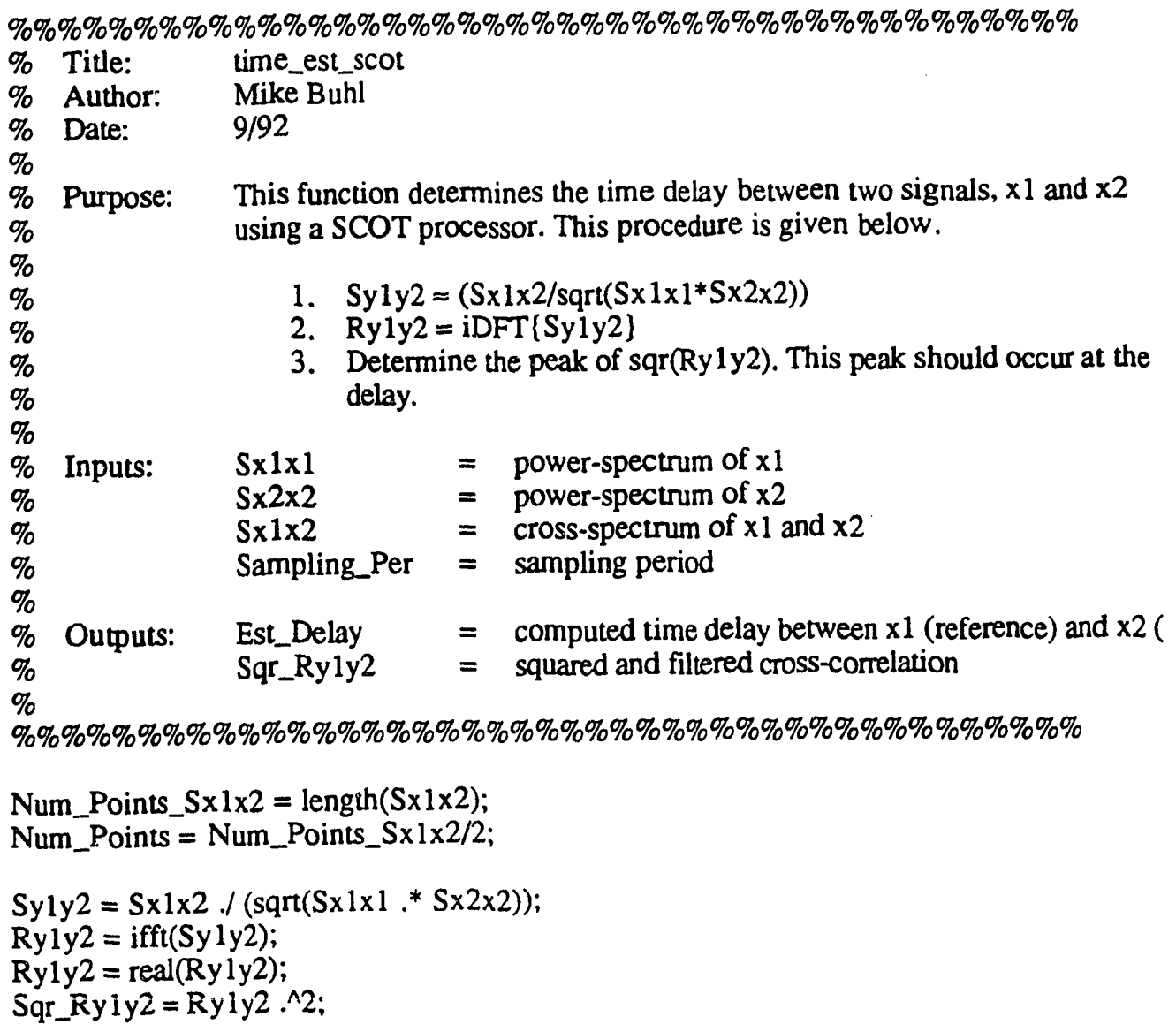




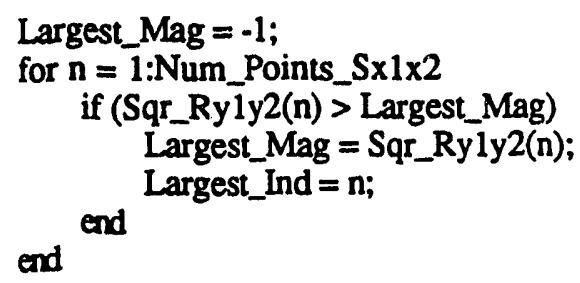

Est_Delay $=\left(\left(\right.\right.$ Largest_Ind-1)-Num_Points) ${ }^{*}$ Sampling_Per,

\section{B.9. time_est_phat.m}

function [Est_Delay, Sqr_Ry 1y2] = time_est_phat(Sx 1x2,Sampling_Per);

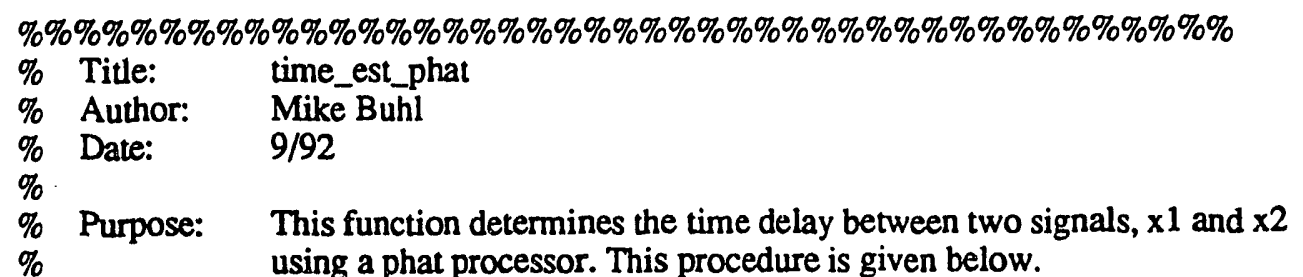

1. $S y 1 y 2=S x 1 \times 2 / a b s(S x 1 \times 2)$

2. Ry1y2=iDFT (Sy1y2)

3. Determine the peak of $s q r(R y 1 y 2)$. This peak should occur at the delay.

$$
\begin{aligned}
& \text { Inputs: } \quad S \times 1 \times 2=\text { cross-spectrum of } x 1 \text { and } \times 2 \\
& \text { Sampling_Per }=\text { sampling period } \\
& \text { Outputs: Est_Delay } \quad=\text { computed time delay between } \times 1 \text { (reference) and } \times 2 \text { ( } \\
& \text { Sqr_Ryly2 = squared and filtered cross-correlation }
\end{aligned}
$$

\%\%\%\%\%\%\%\%\%\%\%\%\%\%\%\%\%\%\%\%\%\%\%\%\%\%\%\%\%\%\%\%\%\%\%\%\%\%\%\%\%\%\%

Num_Points_Sx $1 \times 2=$ length $(\mathrm{S} \times 1 \times 2)$;

Num_Points $=$ Num_Points_S $\times 1 \times 2 / 2$;

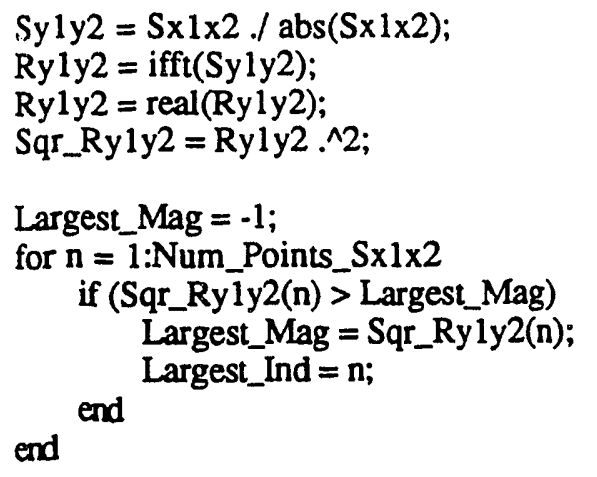

Est_Delay $=\left((\right.$ Largest_Ind-1 $\left.)-N u m \_P o i n t s\right) *$ Sampling_Per, 


\section{B.10. time_est_ml.m}

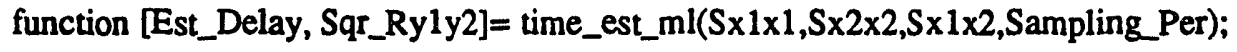

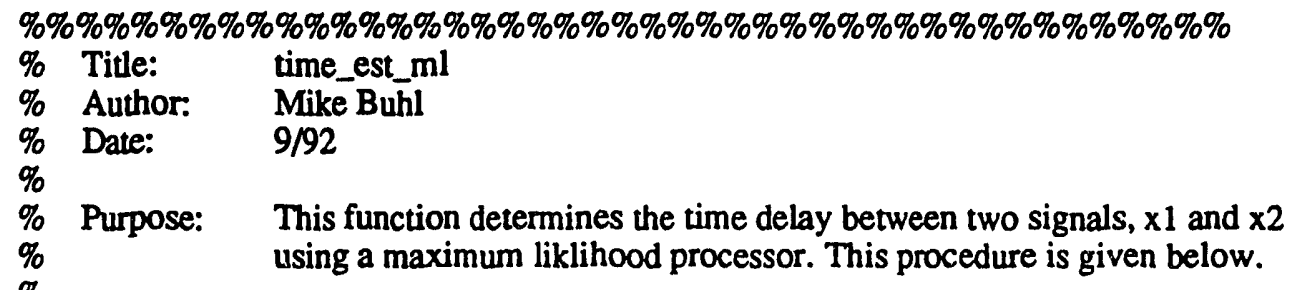

1. $C \times 1 \times 2 \approx S \times 1 \times 2 /(\operatorname{sqrt}(S \times 1 \times 1 * S \times 2 \times 2))$

2. Sy $1 \mathrm{y} 2 \approx(\mathrm{Sx} 1 \times 2 * \mathrm{Cx} 1 \times 2 * \operatorname{conj}(\mathrm{Cx} 1 \times 2)) /(\operatorname{abs}(\mathrm{S} \times 1 \times 2) *$ $(1-\mathrm{Cx} 1 \times 2 * \operatorname{conj}(\mathrm{Cx} 1 \times 2)))$

3. $\mathrm{Ry} 1 \mathrm{y} 2=\mathrm{iDFT}($ Sy1 2 2)

4. Determine the peak of $\operatorname{sqr}(\operatorname{Ry} 1 \mathrm{y} 2)$. This peak should occur at the delay.

Inputs: $\quad$ Sx1 $x 1=$ power-spectrum of $x 1$

$\mathrm{S} \times 2 \times 2=$ power-spectrum of $\times 2$

Sx1 $2=$ cross-spectrum of $x 1$ and $\times 2$

Sampling_Per $=$ sampling period

Outputs: Est_Delay $\quad=$ computed time delay between $\times 1$ (reference) and $\times 2$ (

Sq__Ryly2 = squared and filtered cross-correlation

\%\%\%\%\%\%\%\%\%\%\%\%\%\%\%\%\%\%\%\%\%\%\%\%\%\%\%\%\%\%\%\%\%\%\%\%\%\%\%\%\%\%\%

Num_Points_Sx $1 \times 2=$ length $(S \times 1 \times 2)$;

Num_Points $=$ Num_Points_Sx $1 \times 2 / 2$;

sqr_Cx $1 \times 2=(\operatorname{abs}(\operatorname{Sx} 1 \times 2) . \wedge 2) . /\left(\operatorname{Sx} 1 \times 1 .{ }^{*} \mathrm{~S} \times 2 \times 2\right)$

Sy1y2 = Sx1x2 .*(sqr_Cx1x2./(abs(Sx1x2) .* (1 - sqr_Cx1x2)));

Ry1y2 = ifft(Syly2);

Ry1y2 = real(Ryly2);

Sqr_Ry1y2 = Ry 1 y2.$\wedge 2$;

Largest_Mag $=-1$;

for $n=1$ :Num_Points_Sx $1 \times 2$

if $($ Sqr_Ry1 y2 $(n)>$ Largest_Mag)

Largest_Mag = Sqr_Ryly2(n);

end

Largest_Ind $=\mathrm{n}$;

end

Est_Delay $=(($ Largest_Ind-1)-Num_Points $) *$ Sampling_Per,

\section{B.11. locate_source.m}

function Est_Src_Cor = locate_source(Sen_Cor,Ref_Sen,Est_Delay,Sig_Vel)

\%\%\%\%\%\%\%\%\%\%\%\%\%\%\%\%\%\%\%\%\%\%\%\%\%\%\%\%\%\%\%\%\%\%\%\%\%\%\%\%\%\%\%

$\%$

$\%$ Title: locate_source

$\%$ Áuthor: Míke Buni 


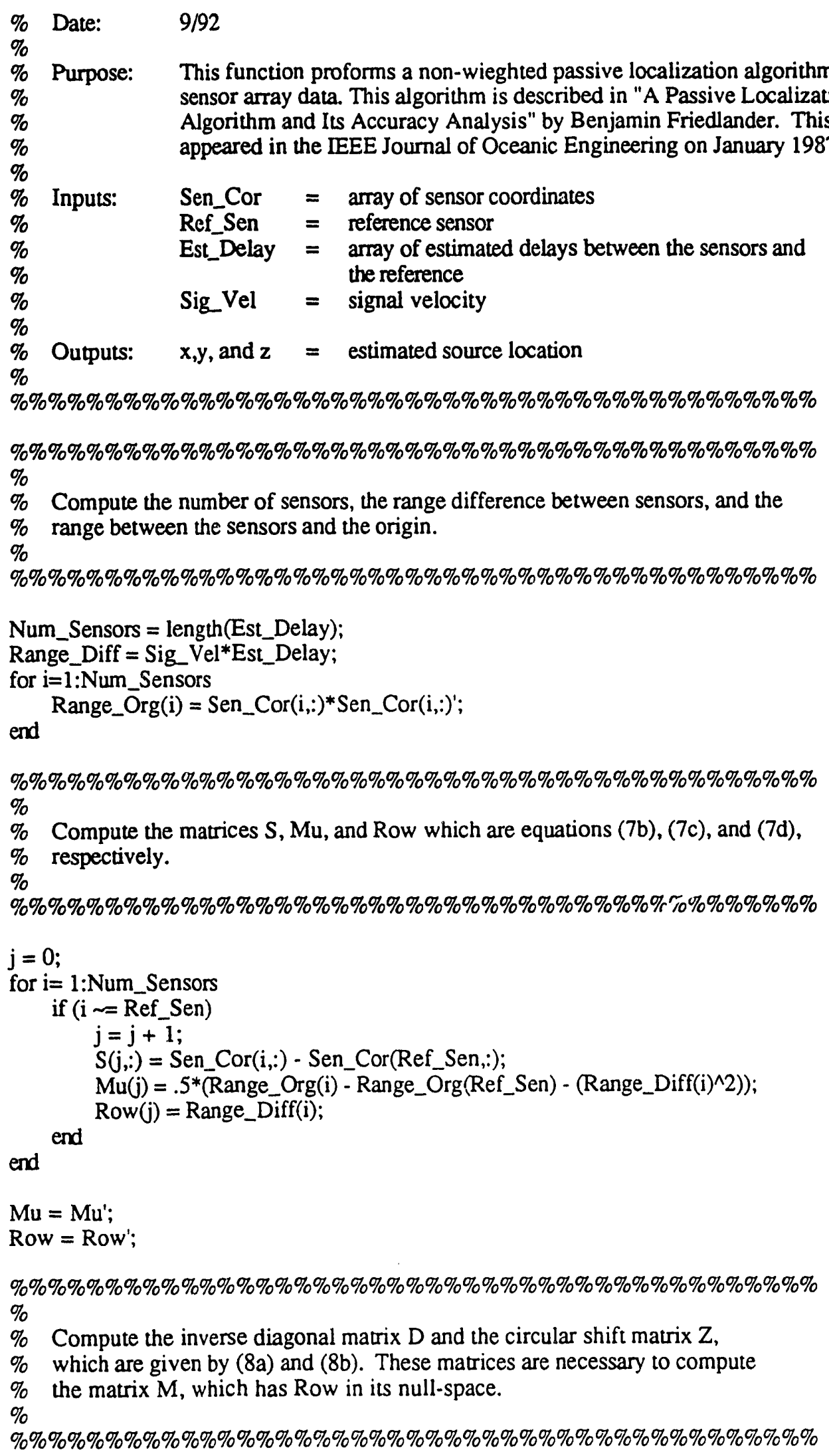




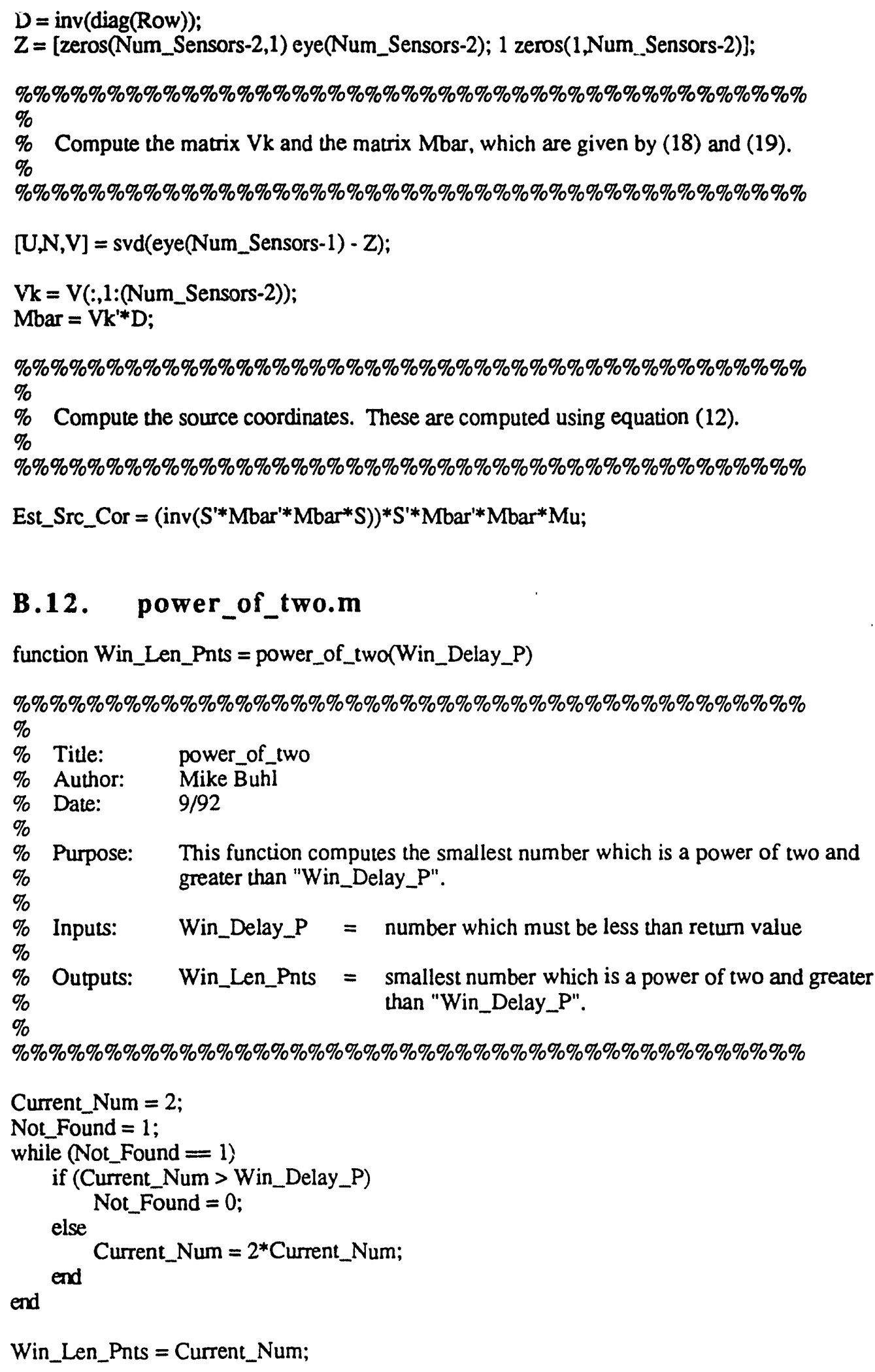



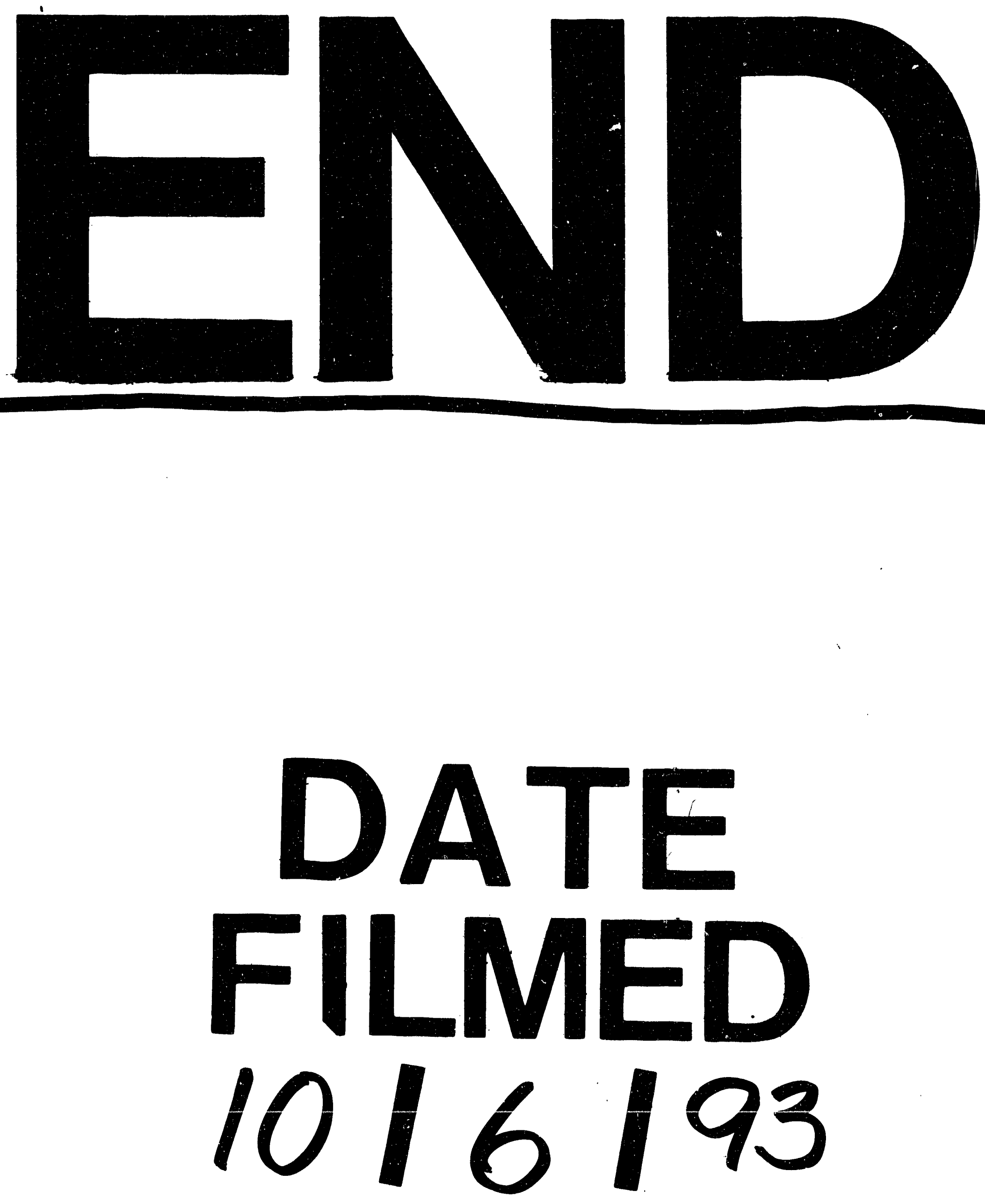